\title{
Simulating the effect of microcracks on the diffusivity and permeability of concrete using a three-dimensional model
}

\author{
S.D. Abyaneh, H.S. Wong ${ }^{*}$ and N.R. Buenfeld \\ Concrete Durability Group, Department of Civil and Environmental Engineering, Imperial College London,
}

$S W 72 A Z, U K$

\begin{abstract}
Concrete inevitably contains microcracks, but their significance on transport properties and long-term durability is not well established. This is because of difficulties in isolating and evaluating the effect of microcracks whether by laboratory experiments or computer simulations, owing to their complex heterogeneous nature. In this paper, a three-dimensional numerical approach to simulate mass transport properties of concrete containing microcracks is presented. The approach is based on finite-element method and adopts aligned meshing to improve computational efficiency. The mesostructure of concrete is represented by aggregate particles that are surface meshed by triangulation and porous cement paste matrix that are discretised with tetrahedral elements. Microcracks are incorporated as interface elements at the aggregate-paste interface or at the cement paste matrix spanning neighbouring aggregate particles. The main advantage of this approach is that the smallest microcracks can be simulated independent of the discretisation size. The model was first validated by comparing the simulations to available analytical solutions. Then, the diffusivity and permeability of a range of concretes containing different amounts of microcracking with increasing complexities were simulated. The results are analysed and discussed in terms of the effect of microcrack type (bond, matrix), volume fraction, width, specific surface area and degree of percolation on transport properties.
\end{abstract}

Keywords: Concrete; Mortar; Cement-based materials; Diffusivity; Permeability; Microcrack.

\section{Introduction}

The long-term durability of concrete structures is largely dependent on the resistance of the concrete to penetration of aggressive species (e.g. chloride, $\mathrm{CO}_{2}$, sulphates, water) when exposed to its service environment. This is because most degradation mechanisms affecting concrete are rate-controlled by the transport of aggressive species through its inevitably porous microstructure. For example, the service-life of concrete structures in a marine environment is often determined by the time it takes for chloride ions to penetrate the concrete cover in sufficient quantities to induce corrosion of embedded steel reinforcement. The main transport mechanisms occurring in concrete and generally in any porous medium are diffusion, permeation and absorption. The understanding of transport processes in concrete, and how various phases in its microstructure influence transport, is absolutely critical for the development of more durable and sustainable concretes.

In practice, concrete structures are subjected to various types of actions that induce tensile stresses exceeding the tensile strength of concrete, causing cracks to form [Concrete Society, 2010]. These include structural loading, thermal gradients, wet/dry cycles and freeze/thaw cycles. As such, concrete structures in service are almost always cracked. Cracks much wider than $0.1 \mathrm{~mm}$ cause leakage and affect the watertightness of concrete structures. Cracks also act as pathways for aggressive agents, thereby accelerating deterioration [Concrete Society, 1995]. When the cracks percolate, their influence on transport far outweighs that of capillary pores because of their larger size and shorter flow lengths. Therefore, cracks not only affect watertightness, but also long-term durability of concrete structures.

Cracks larger than $0.1 \mathrm{~mm}$ can be controlled and eliminated by proper design and placement of embedded steel reinforcements. However, cracks smaller than $0.1 \mathrm{~mm}$, i.e. "microcracks", are much harder to control and eliminate via structural design alone. It has long been suspected that microcracks, regardless

\footnotetext{
* Corresponding author. E-mail address: hong.wong@imperial.ac.uk. Telephone: +44 (0)20 75945956
} 
of their origins, act as preferential paths for transport and so they may accelerate degradation processes. However, the significance of their influence on bulk transport and durability of concrete is not well understood. This is mainly because of the fact the microstructure of concrete is physically and chemically complex, multi-phase and multi-scale. The microcracks themselves are heterogeneous and spatially variable. Several phases in concrete contribute to transport and the overall property is influenced by many interacting effects, some of which are very difficult to isolate and quantify from laboratory experimentation. For example, one can produce and test concretes with varying amounts of microcracking by subjecting samples to controlled drying. However, this inevitably changes the concrete moisture content and degree of saturation, which also have huge influences on the measured transport properties. In this respect, numerical modelling could provide a more effective and systematic means to study these effects.

Many previous studies have examined the influence of cracks on mass transport properties of concrete such as the work of Jacobsen et al. [1996], Wang et al. [1997], Gerald et al. [1997, 2000], Aldea et al. [1999], Kamali-Bernard and Bernard [2009], Picandet et al. [2009], Grassl et al. [2010], Jang et al. [2011], Akhavan et al. [2012] and Djerbi et al. [2008, 2013]. However, the majority of the available studies relate to large $(>0.1 \mathrm{~mm})$ traversing cracks that go through the entire thickness of the sample. The transport properties of a medium containing such cracks can be modelled in a relatively straightforward manner because the effective property can be related to crack characteristics such as width and area using an analytical parallel model. Furthermore, most modelling work on cracks in concrete has been carried out in two-dimensions. Comparatively few studies have investigated the effect of microcracks $(<0.1 \mathrm{~mm})$ that are randomly dispersed in a three-dimensional microstructure. Such microcracks do not necessarily propagate through the entire thickness; in fact they are discontinuous and are more representative of those induced by shrinkage and thermal effects.

Because of the inherent limitations of analytical models, many researchers have employed numerical homogenization schemes such as finite element, finite difference, random walk and Lattice Boltzmann for investigating transport behaviour in cement-based materials [Garboczi \& Bentz, 2001; Kamali-Bernard, 2009; Abyaneh et al., 2013a, 2013b, 2014; Zalzale \& McDonald, 2012; Zhang et al., 2013; Du et al., 2014; Liu et al., 2015; Feng et al., 2016]. These methods are all very well-known approaches used in many fields to solve differential equations that govern physical behaviours, but application to study the effect of microcracks is limited. A major advantage of these techniques is that they can be explicitly coupled with digital images of the actual microstructure as input. For example, each voxel in a digital image can be considered as an element in finite element simulations. Therefore, a total and direct transference of information between the digital image and the simulation grid can be carried out [Moreno-Atanasio et al., 2010]. However, the main disadvantage of these approaches is that the size of the numerical sample and resolution (voxel size) at which one could simulate is constrained by available computational resources. Thus, the smallest feature that can be modelled realistically is limited. This presents a major challenge for simulating the effect of microcracks in concrete because it is too computationally demanding to model a representative volume of concrete at sufficiently high resolution to capture the microcracks, in threedimension.

An approach to overcome this obstacle is described in this study. The approach is based on aligned meshing whereby aggregate particles and porous cement paste matrix are discretised using tetrahedral elements and the surfaces of aggregate particles are triangulated, enabling these to be represented more accurately and efficiently. This has the advantage over regular meshing using cubic voxels, which inevitably represent curved surfaces as perpendicular planar surfaces. Once the three-dimensional mesostructure of concrete is established, microcracks are then incorporated as interface elements that propagate at the aggregate-paste interface (bond microcracks) and cement paste matrix spanning nearest neighbouring aggregate particles (matrix microcracks). The microcracks can be assigned with unique widths, lengths, orientation and connectivity. The advantage of this approach is that the smallest microcracks can be simulated independent of the discretisation size of the mesostructure. This approach, which was presented in a preliminary study [Abyaneh et al. 2015], will be fully developed and tested in this paper. First, the model is validated by examining ideal cases and comparing simulations to analytical solutions. Then, the model is used to simulate the diffusivity and permeability of a range of concretes containing different amounts of microcracking with increasing complexities. 


\section{Methodology}

\subsection{Approach}

An input structure of the material coupled with a transport algorithm is required in order to model mass transport phenomena. Therefore, our approach is to first generate a three-dimensional mesostructure of concrete where it is idealised as a composite of aggregate particles embedded in a porous cement paste matrix that contains microcracks. The mesostructure will explicitly account for microcracks that propagate around aggregate particles and microcracks that propagate through the cement paste matrix. Other phases such as the interfacial transition zone and air voids may also be included, but these features are not the focus of this study and so will be ignored. The mesostructure is then meshed using an aligned meshing approach and the microcracks are incorporated as either bond or matrix microcracks with different widths, lengths, orientations and degrees of connectivity. Each element within the mesostructure is assigned a transport property according to the phase that it represents. A finite element method is then applied to the entire mesostructure to simulate bulk diffusion and permeation at steady-state conditions.

\subsection{Mesostructure}

To generate a mesostructure of concrete, spherical aggregate particles were placed randomly in a computational cube. The aggregate particles have varying sizes ranging from $1 \mathrm{~mm}$ to $10 \mathrm{~mm}$, and follow the Fuller-Thompson gradation. Total aggregate volume fraction ranged from $10 \%$ to $60 \%$. An earlier study [Abyaneh et al., 2013a] showed that a computational cube that is 2.5 times the largest aggregate particle can give representative results if sufficient number of replicates is simulated and the results averaged. Aggregate particles were placed in the order of descending size with no overlaps. However, periodic boundary conditions were not applied to avoid potential meshing problems during the aligned meshing process. The non-periodic boundary conditions could produce preferential edge flow and this could distort overall results. However, our simulations (presented later) show that this effect is small and negligible for the sample size and resolution used in this study. For a $25 \mathrm{~mm}$ cube containing $60 \%$ vol. of aggregate particles ranging from 1 to $10 \mathrm{~mm}$, around 1000 particles were required. Examples of the generated concrete mesostructure are shown in Fig. 1.

A finite element package (ABAQUS) was used to mesh the concrete mesostructure via aligned meshing. Surfaces of the aggregate particles were first triangulated, and then the aggregate particles and porous cement paste matrix were discretised using tetrahedral elements. This process is shown in Fig. 2(a) and 2(b). For the sake of clarity, a relatively coarse mesh of $1 \mathrm{~mm}$ global element size (i.e. spacing between nodes) is shown in Fig. 2. A much finer global element size of $0.25 \mathrm{~mm}$ was used in the actual transport simulations and each mesostructure contained $\sim 6.5 \times 10^{6}$ tetrahedral elements. The frequency distributions of the tetrahedral element volume and normalised shape factor for a mesostructure containing $60 \%$ vol. aggregate particles are shown in Fig. 3. The observed narrow distribution of the element volume is an important feature of the model and shows that the generated mesh is of a high quality and should lead to reliable results at a lower computational expense. The normalised shape factor is defined as the ratio of element volume to the optimal element volume, where the latter is the volume of an equilateral tetrahedron with the same circumradius as the element. The normalised shape factor should range from 0 to 1 , with " 1 " indicating an optimal element shape and " 0 " indicating a degenerated element. Thus, the distribution of shape factor also lends support that the mesh is of high quality.

Since most aggregate particles used in concrete are dense compared to the cement paste, it is assumed that the aggregate particles have zero transport property and flow occurs only through the interconnected cement paste matrix. Thus, it would be computationally beneficial to remove the tetrahedral elements representing the aggregate particles. In doing so, the number of elements decreases from $\sim 6.5 \times 10^{6}$ to $\sim 3.3 \times$ $10^{6}$ and the number of nodes reduces from $\sim 1.1 \times 10^{6}$ to $\sim 6.9 \times 10^{5}$ for a typical sample containing $60 \%$ vol. aggregate particles. It should be noted that the number of nodes is equal to the degrees of freedom of the system as the concentration value should be calculated at each node (one scalar value at each node). Fig. 3 and Fig. 4 show the effect of this on the frequency distribution of element volume and shape factor. The results show that the quality of the mesh is maintained after removal of the elements embedded in aggregate particles and for different realisations regardless of the aggregate volume fraction. 
(a)

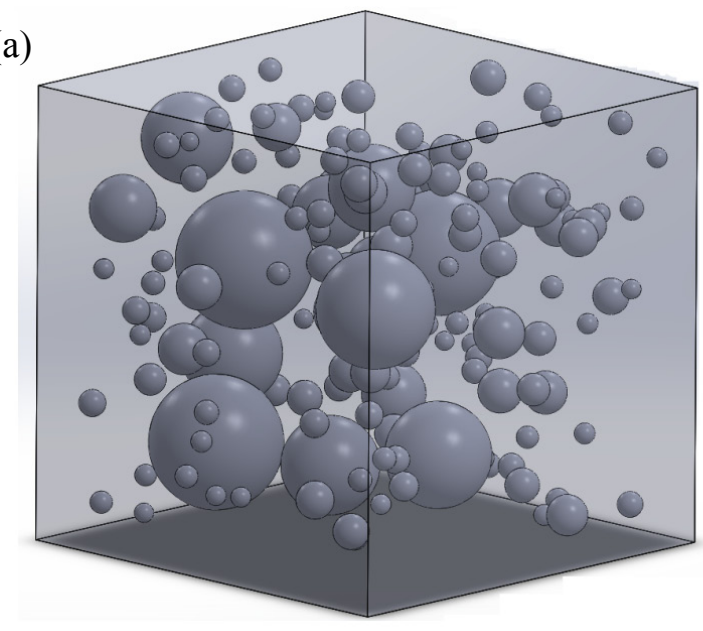

(c)

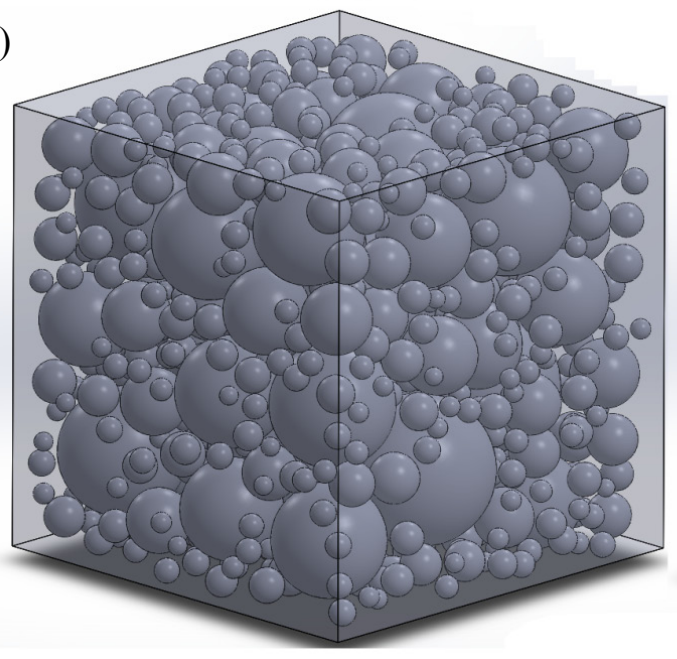

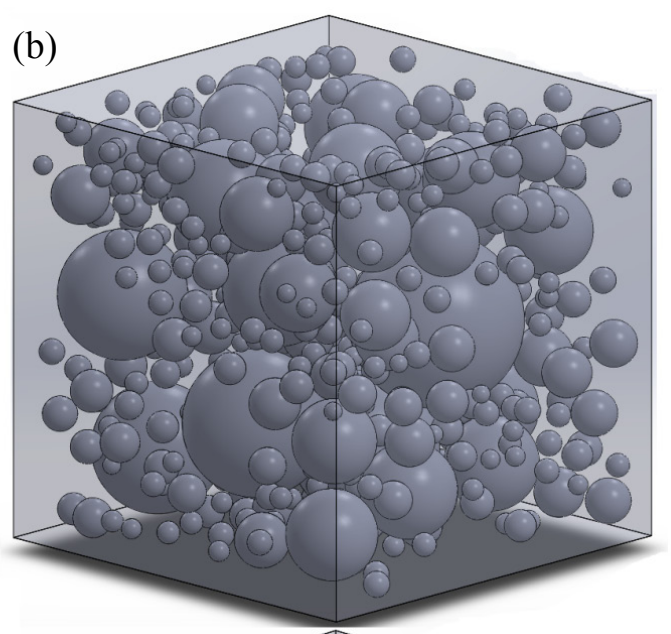

(d)

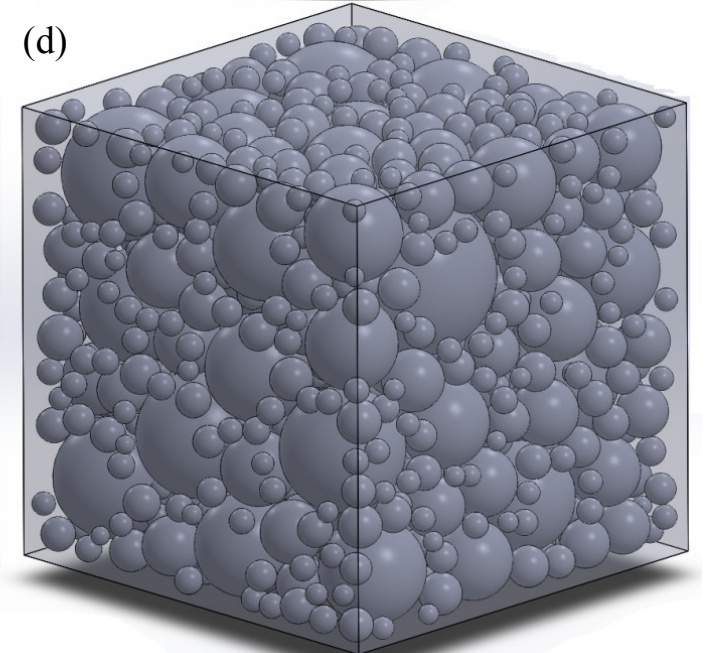

Fig. 1 Mesostructure of concrete generated by randomly placing aggregate particles ranging from 1 to $10 \mathrm{~mm}$ (Fuller-Thompson gradation) in a $25 \times 25 \times 25 \mathrm{~mm}^{3}$ computational cube. Total aggregate particle volume fractions are: a) $10 \%$, b) $30 \%$, c) $50 \%$ and d) $60 \%$.

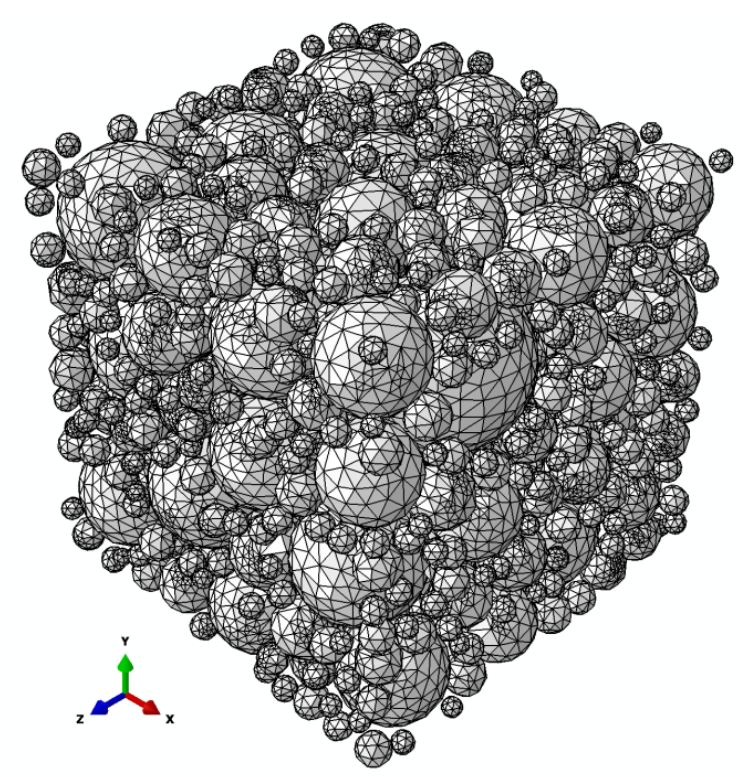

(a)

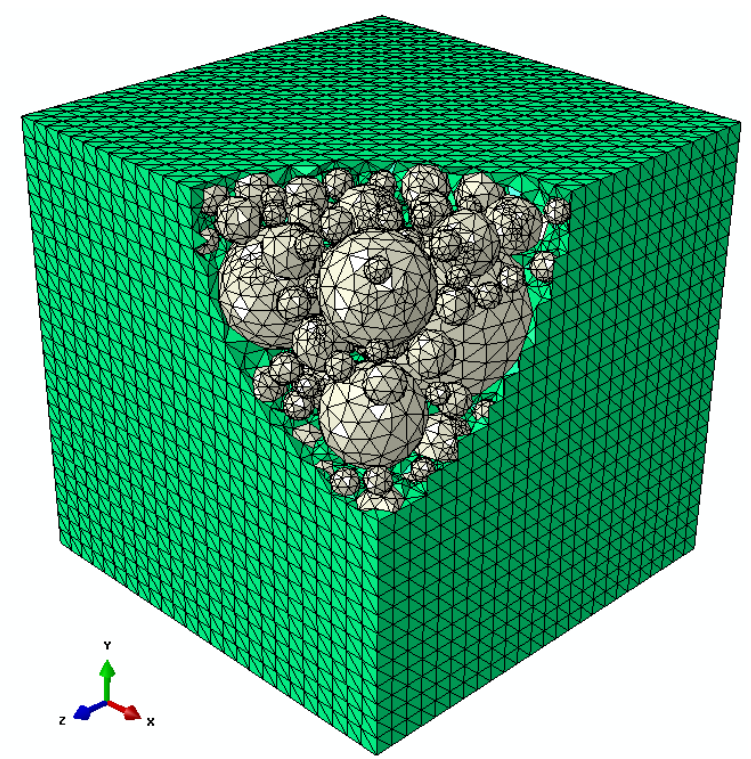

(b)

Fig. 2 Demonstration of the aligned meshing procedure. Surfaces of aggregate particles are first meshed by triangulation (a) and then the cement paste matrix is filled with tetrahedral elements (b). This example is a mesostructure containing $60 \%$ vol. aggregate particles in a $25 \times 25 \times 25 \mathrm{~mm}^{3}$ computational cube [Abyaneh et al. 2015]. 


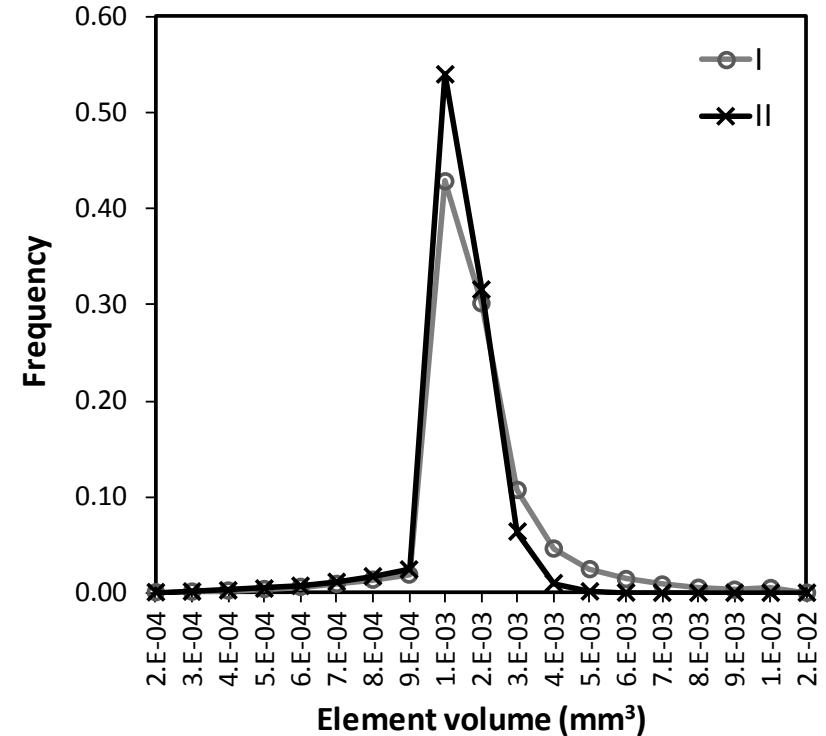

(a)

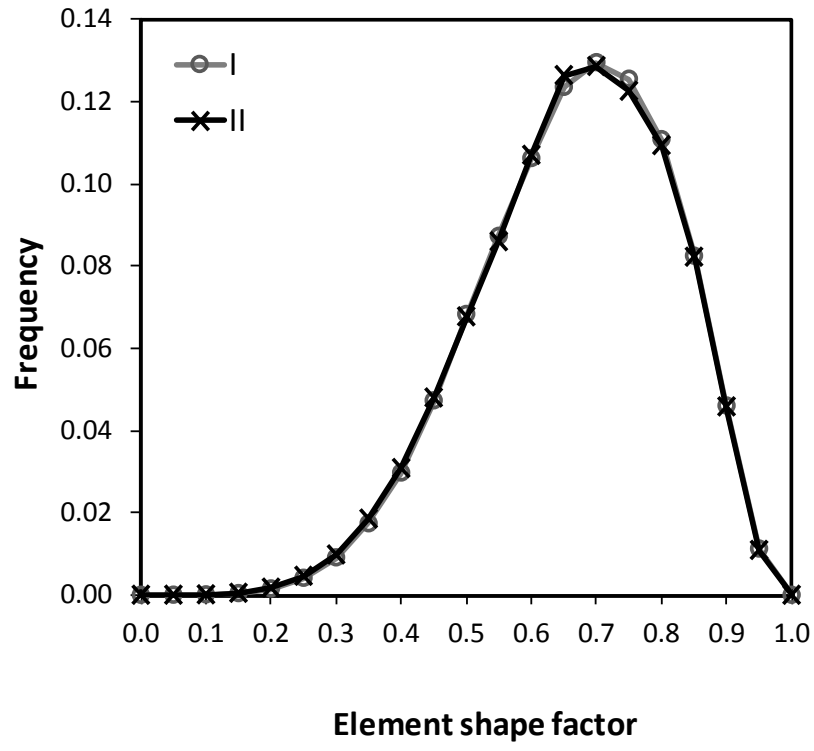

(b)

Fig. 3 Frequency histograms of the tetrahedral element volume and shape factor for the concrete mesostructure containing 60\% vol. aggregate particles shown in Fig. 2. Mesh (I) contains $\sim 6.5 \times 10^{6}$ tetrahedral elements with $\sim 1.1 \times 10^{6}$ nodes (degrees of freedom). In mesh (II), the elements embedded within aggregate particles are removed to increase computational efficiency. This reduces the number of elements to $\sim 3.3 \times 10^{6}$ with $\sim 6.9 \times 10^{5}$ nodes.

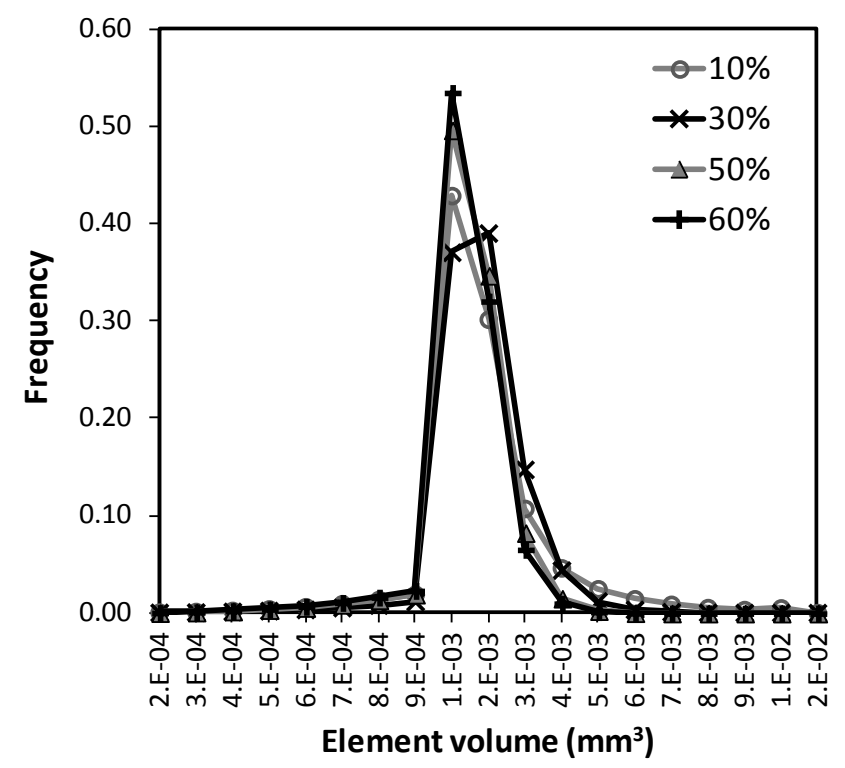

(a)

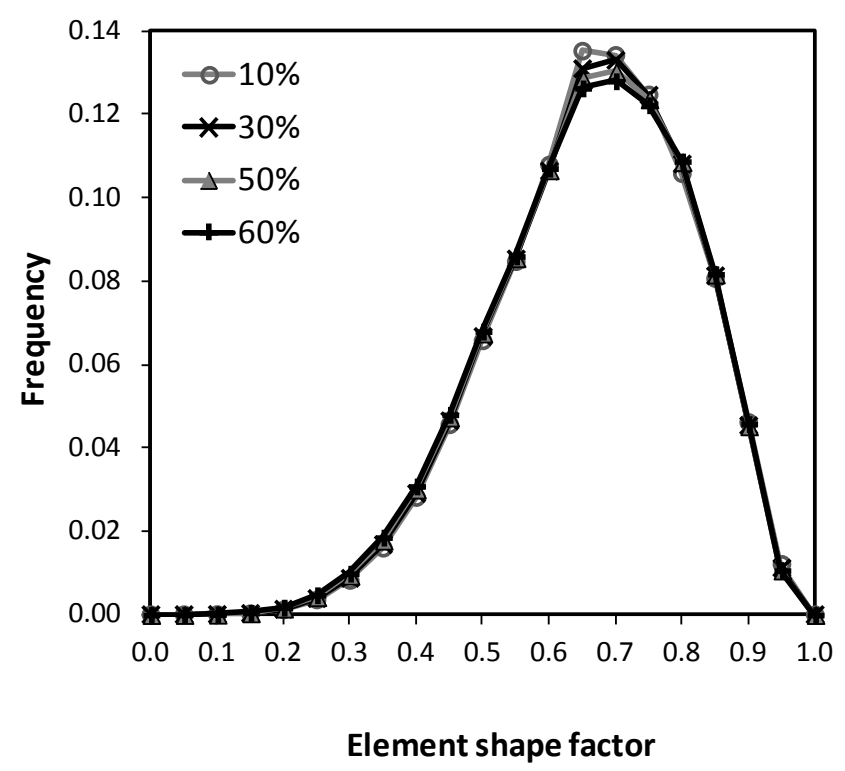

(b)

Fig. 4 Frequency histograms of the tetrahedral element volume and shape factor for concrete mesostructures containing $10 \%, 30 \%, 50 \%$ and $60 \%$ vol. aggregate particles. Elements embedded within aggregate particles were removed. Total number of elements ranged from 3.3 to $4.7 \times 10^{6}$. 


\subsection{Input structure containing microcracks}

The microcracks are incorporated as interface elements; these are triangulated during the meshing process and each microcrack is assigned its own characteristics such as width, length and orientation. As mentioned earlier, the main advantage of this is that the microcracks can be represented independently of the size of discretisation of the aggregate particles and cement paste matrix. The microcracks will have widths much smaller than the dimensions along the other two axes. Therefore, each microcrack is approximately a two-dimensional plane.

In order to study the effect of microcracks on transport properties, samples containing different microcrack patterns with increasing complexities were implemented. First, simple crack patterns such as parallel orthogonal cracks that traverse through the thickness of the mesostructure (Fig. 5a) were simulated and compared to analytical solutions as a means of validation. Then, mesostructures containing bond microcracks were simulated by either placing bond microcracks in a homogeneous medium (Fig. 5b) or in a heterogeneous medium containing aggregate particles and cement paste (Fig. 5c). In the simulations, microcrack volume fraction was increased by increasing the width of the microcracks in a mesostructure containing $60 \%$ vol. aggregate particles $(1-10 \mathrm{~mm})$. Microcracks with widths of 1, 5, 10, 25 and $50 \mu \mathrm{m}$ were assumed.

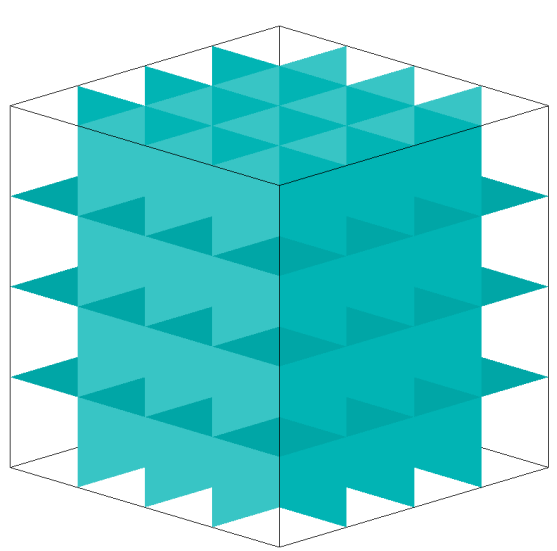

(a)

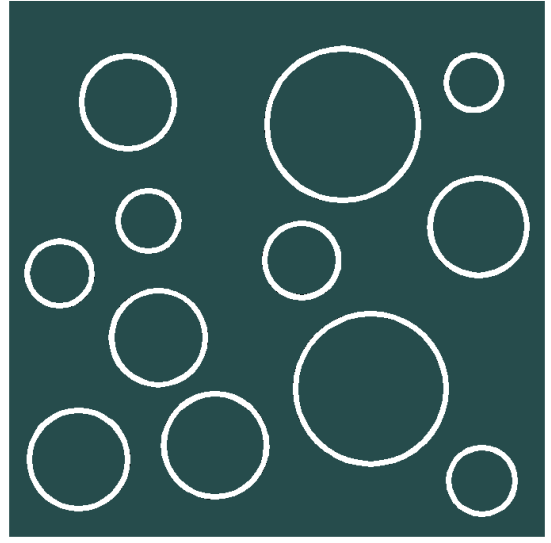

(b)

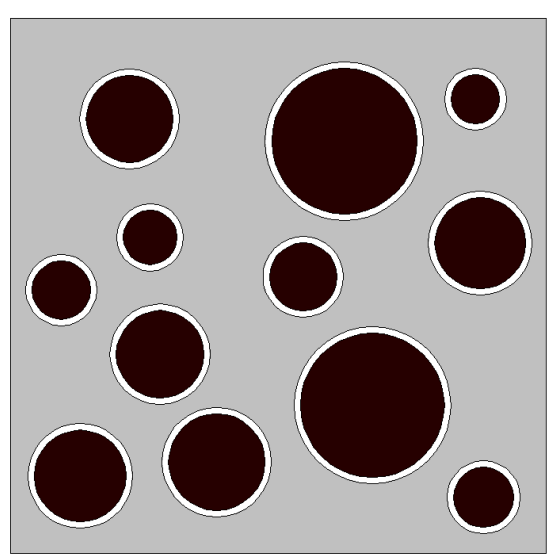

(c)

Fig. 5 Schematics showing a parallel orthogonal crack network (a) and bond crack networks (b, c). In (b), bond cracks are dispersed in a homogenised media while in (c) bond cracks are dispersed in a heterogeneous media containing aggregate particles and cement paste.

Subsequently, more realistic microcrack patterns consisting of bond and matrix microcracks were implemented. Six microcrack networks with increasing degree of microcracking were generated on a concrete mesostructure containing $60 \%$ vol. aggregate particles. It is absolutely critical that the size, amount and distribution of the microcracks should be such that the generated networks mimic actual cracks in concrete as much as possible. The exact crack pattern is difficult to predict theoretically. However, it can be shown that cracks will occur in regions of high stress concentrations, propagate through the shortest path to release stresses, or through the weakest phases, or follow a path combining these criteria [Goltermann, 1994; 1995]. It is well established that microcracks often initiate at the weak interface between aggregate particles and cement paste matrix, predominantly on large aggregate particles, and then propagate along the aggregate-paste interface or across the cement paste matrix to span neighbouring aggregate particles [Hsu, 1963; Slate \& Olsefski, 1963; Shah \& Chandra, 1968; Goltermann 1994, 1995].

On this basis, the microcrack network was generated by distributing bond microcracks around randomly selected large aggregate particles. Then, matrix microcracks were placed to span the nearest neighbouring aggregate particles, i.e. via the smallest separating distances first. In other words, an algorithm identifies the nearest distances (in three-dimensions) between aggregate particles and then connects these particles with triangular interface elements representing the matrix microcracks. This procedure is schematically represented in two-dimension in Fig. 6 . The amount of bond and matrix microcracks were gradually increased until the first microcrack network achieved a microcrack specific surface area, i.e. microcrack surface area per sample volume, of $0.15 \mathrm{~mm}^{2} / \mathrm{mm}^{3}$. The process was repeated to produce six microcrack networks ( $a$ to $f$ ) with increasing degree of microcracking, up to a microcrack density of $0.90 \mathrm{~mm}^{2} / \mathrm{mm}^{3}$. Table 1 shows the number of bond microcracks, matrix microcracks and specific surface area of the six 
microcrack networks. Note that the number of microcracks and specific surface area were increased at a regular interval. The ratio of matrix to bond microcracks was kept at a fairly constant value of between 1.24 and 1.28 .

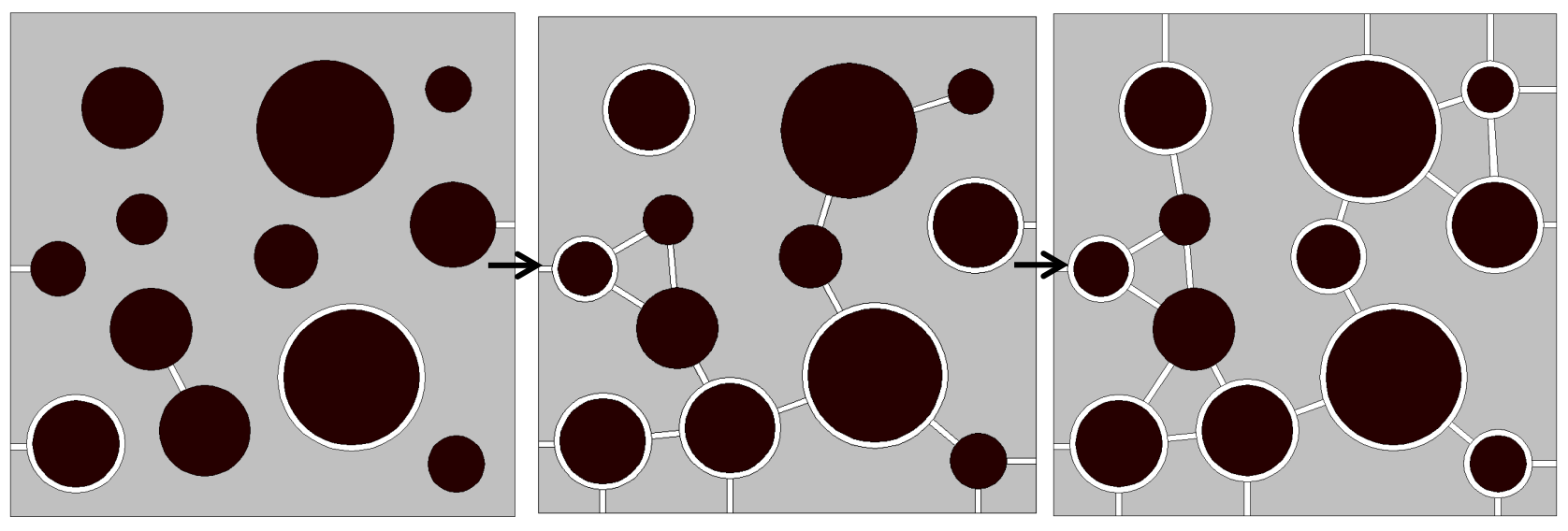

Fig. 6 Schematic of two-dimensional sections showing the generation of bond and matrix microcrack networks with increasing degree of microcracking. Bond microcracks are placed around randomly selected aggregate particles while matrix microcracks are placed to span nearest neighbouring aggregate particles [Abyaneh et al., 2015].

Table 1 Characteristics of the generated bond and matrix microcrack networks. Microcracks were assigned widths of $1,5,10,25$ or $50 \mu \mathrm{m}$ to produce a range of microcrack volume fraction.

\begin{tabular}{lcccccc}
\hline & \multicolumn{7}{c}{ Microcrack network } \\
\cline { 2 - 7 } Property & $a$ & $b$ & $c$ & $d$ & $e$ & $f$ \\
\hline Number of bond microcracks & 154 & 298 & 452 & 606 & 750 & 904 \\
Number of matrix microcracks & 191 & 382 & 573 & 765 & 956 & 1147 \\
Microcrack volume fraction (\%) & $0.02-0.75$ & $0.03-1.45$ & $0.04-2.15$ & $0.06-2.90$ & $0.07-3.70$ & $0.09-4.50$ \\
Specific surface area $\left(\mathrm{mm}^{2} / \mathrm{mm}^{3}\right)$ & 0.15 & 0.29 & 0.43 & 0.58 & 0.74 & 0.90 \\
Percolation & No & No & No & No & Yes & Yes \\
\hline
\end{tabular}

A burning algorithm [Stauffer, 1985] was used to determine the degree of percolation of the generated microcrack networks. This was done by simulating the overall transport property of the sample as the transport property of the microcracks was increased to infinity. If the simulation shows that the overall transport property of the sample also tended to infinity, then it can be deduced that microcrack network was percolated. On this basis, it was concluded that the first four microcrack networks $a, b, c$ and $d$ are not percolated, while the last two microcrack networks $e$ and $f$ are percolated. Another approximation was the width of the microcracks. These were assigned equal values of 1, 5, 10,25 or $50 \mu \mathrm{m}$ for the sake of simplicity. Therefore, the microcrack volume fraction for all the simulated mesostructures ranged between $0.015 \%$ and $4.5 \%$, as calculated by multiplying the specific surface area with the microcrack width. Despite these approximations, it is noted that the simulated microcrack width, volume fraction and specific surface area are well within the range that have been measured for drying shrinkage-induced microcracks in concrete [Wong et al., 2009; Wu et al., 2014; 2015]. 


\subsection{Governing equations: diffusion}

Diffusion through the cracked medium was modelled using finite element method by considering the diffusivities of the porous cement paste matrix, aggregate particles and cracks. However, the aggregate particles typically used in concrete are dense compared to the cement paste matrix. Therefore, the elements representing the aggregate particles can be assigned zero diffusivity and are assumed to have no contribution to flow. According to Fick's first law of diffusion, the local flux $\bar{J}_{m}$ is written as:

$$
\bar{J}_{m}=-D_{m} \nabla c
$$

Where $D_{m}$ is the diffusivity of the cement paste matrix and $c$ is the concentration of the diffusing species. At steady-state condition, the conservation of local fluxes in the porous matrix can be expressed as: $\nabla \cdot \bar{J}_{m}=0$. Similarly, the flux through a crack can be written in the following form:

$$
J_{c r}=-w_{c r} D_{c r} \nabla_{s} c
$$

Where $w_{c r}$ is the crack width and $D_{c r}$ is the diffusivity of the crack, the latter can be taken to be equal to the free diffusivity $D_{o}$,

This set of differential equations is then solved by the finite element method, and solutions are averaged on a larger scale by integrating the local fluxes over the porous matrix and crack surfaces, divided by the total volume of the media. This gives the total flux $\overline{\bar{j}}$ :

$$
\overline{\overline{\boldsymbol{J}}}=\frac{1}{\tau_{0}}\left(\int_{\tau_{m}} \bar{J}_{m} d \tau+\int_{S_{c r}} \boldsymbol{J}_{c r} d s\right)
$$

Where $\tau_{\mathrm{o}}, \tau_{\mathrm{m}}$ and $S_{c r}$ are the sample volume, matrix volume and crack surface area respectively. Note that total flux is related to the concentration gradient via:

$$
\overline{\overline{\boldsymbol{J}}}=-D_{e f f} \overline{\nabla c}
$$

For the general case of an anisotropic medium, $D_{\text {eff }}$ is a tensor. However, if the concentration gradient is imposed in one direction and other surfaces are assumed impermeable, the two last equations are simplified to:

$$
\overline{\bar{J}}_{x}=\frac{1}{\tau_{0}}\left(\int_{\tau_{m}} \bar{J}_{m x} d \tau+\int_{S_{c r}} J_{c r x} d s\right)=-D_{e f f} \frac{\overline{\overline{\partial c}}}{\partial x}
$$

Therefore, all nodes on the inlet and outlet surfaces of the computational cube were assigned fixed concentration values, while nodes on the other four boundaries were treated as sealed surfaces allowing no flux through.

\subsection{Governing equations: permeation}

Here, it is assumed that pressure-induced flow though a cracked media can be characterised by applying Darcy's law at the mesoscale and Navier-Stokes equation at the microscale [Adler et al., 2012; Bogdanov et al., 2003; Sahimi, 2011]. Assuming that the porous cement paste matrix has a bulk permeability $K_{m}$ that can vary with position, the local fluid velocity $\bar{v}$ in the matrix is $\bar{v}=-K_{m} \nabla p / \mu$, where $\mu$ is the fluid viscosity and $p$ is pressure. The conservation for local fluid velocity can be written as $\nabla \cdot \bar{v}=0$. Similarly, the flow through each crack is given by a two-dimensional Darcy's law:

$$
J=-\frac{w_{c r} K_{c r}}{\mu} \nabla_{s} p
$$


Where $w_{c r}$ is the crack width and $K_{c r}$ is the permeability of the crack. The permeability of the crack can be obtained from the equation $K_{c r}=w_{c r}^{2} / 12$, for the case of a smooth crack with constant cross-section.

As before, the set of differential equations are solved using finite element method by imposing a pressure gradient along one axis and assuming all other surfaces to be impermeable. Subsequently, the solutions are averaged on a larger scale by integrating the flow rates over the porous matrix and crack surfaces divided by the total volume of the medium to obtain the overall fluid velocity $\overline{\bar{v}}$ :

$$
\overline{\bar{v}}=\frac{1}{\tau_{0}}\left(\int_{\tau_{m}} \overline{\boldsymbol{v}} d \tau+\int_{S_{c r}} \boldsymbol{J} d s\right)
$$

Where $\tau_{0}$ is the sample volume, $\tau_{\mathrm{m}}$ is the matrix volume and $S_{c r}$ is the crack surface area as before. Note that the overall fluid velocity is related to the pressure gradient by Darcy's law: $\overline{\bar{v}}=-K_{e f f} \overline{\nabla p} / \mu . K_{\text {eff }}$ is a tensor for the general case of an anisotropic medium. But because pressure gradient is imposed in one direction and other surfaces are assumed to be impermeable, the above equation can be simplified as:

$$
\overline{\bar{v}}_{x}=\frac{1}{\tau_{0}}\left(\int_{\tau_{m}} \bar{v}_{x} d \tau+\int_{S_{c r}} J_{x} d s\right)=-\frac{K_{e f f}}{\mu} \frac{\overline{\overline{\partial p}}}{\partial x}
$$

Therefore, constant pressure values were applied to all nodes on the inlet and outlet surfaces of the computational cube while nodes on the other four boundaries were treated as sealed surfaces allowing no flux through.

Once the mesh is generated, this set of differential equations is solved using the finite element package ABAQUS. It is noted that that in the current implementation, cracks are superimposed on the matrix and not substituted. This means that the cracks are incorporated by superimposing the interface elements without decreasing the volume of adjacent tetrahedral elements. Consequently, the volume of the cracked sample will be increased by a volume equal to that of the cracks. This procedure reduces computational problems and any artefacts caused by it are negligible because the transport property of the added element (crack) is dominant.

As previously stated, the tetrahedral elements within the aggregate particles were removed to decrease computational cost. Technically, removing the tetrahedral elements within the aggregate particles will lead to the rows and columns representing the nodes within the aggregate particle to be removed from the assembled global stiffness matrix in finite element calculations. This process is equivalent to the meshing of aggregate particles and assigning zero transport property (diffusivity/permeability) to them.

\section{Results}

\subsection{Size, resolution and statistical effects}

Any numerical model is inevitably subjected to effects of finite sample size, resolution and statistical fluctuation. Size effects arise because the numerical sample size has been limited to increase computation speed, while statistical fluctuations occur because of the random nature of the model. For example, the placement of aggregate particles is carried out randomly and so the resultant mesostructure is different at each realisation. As such, it is important to determine the requirements in terms of resolution, sample size and number of realisations to obtain representative results. In order to study these effects, the diffusivity of a sample with $60 \%$ vol. aggregate particles was simulated at increasing resolution by changing the global element size from $0.5 \mathrm{~mm}$ to $0.25 \mathrm{~mm}$ and $0.125 \mathrm{~mm}$. In addition, the simulation was carried out for four different realisations of a sample with $60 \%$ vol. aggregate particles as shown in Fig. 7a.

Table 2 shows the effect of resolution on the simulated diffusivity ratio $D / D_{c p}$ along the three principal axes. The results show that the simulated diffusivity ratio changes only very slightly with increase in resolution and that the difference between the simulated values at $0.25 \mathrm{~mm}$ and $0.125 \mathrm{~mm}$ element size was 
no more than $1.6 \%$. Fig. 7 presents the frequency distributions of the tetrahedral element volume and normalised shape factor for the four different realisations of a mesostructure containing $60 \%$ vol. aggregate particles. It can be seen that the obtained frequency distributions are nearly identical and that the quality of the generated mesh is maintained for replicate mesostructure with variation in aggregate particle placement. The results presented in Table 3 shows that any statistical fluctuations produce only a very small difference in the simulated diffusivity. Because aggregate particles are placed randomly in the computational cube, the tested mesostructures are expected to exhibit isotropic behaviour, so the diffusivity along the three principal axes should be identical and can be represented as a single scalar value. The data presented in Tables 2 and 3 show that this is indeed the case. It should also be noted that no difference was observed when the size of the computational cube was increased from $25 \times 25 \times 25 \mathrm{~mm}^{3}$ to $30 \times 30 \times 30 \mathrm{~mm}^{3}$. These results show that the effect of neglecting periodic boundary conditions in the mesostructure is small and negligible.

Table 2 Effect of resolution (global element size) on the simulated diffusivity ratio $D / D_{c p}$ along the three principal axes for a sample containing $60 \%$ aggregate particle volume fraction.

\begin{tabular}{lccc}
\hline \multirow{2}{*}{ Diffusivity ratio } & \multicolumn{3}{c}{ Global element size (mm) } \\
\cline { 2 - 4 } & 0.5 & 0.25 & 0.125 \\
\hline$D_{x} / D_{c p}$ & 0.321 & 0.306 & 0.301 \\
$D_{y} / D_{c p}$ & 0.320 & 0.305 & 0.300 \\
$D_{z} / D_{c p}$ & 0.320 & 0.305 & 0.300 \\
\hline
\end{tabular}

Table 3 Effect of statistical fluctuation on the simulated diffusivity ratio $D / D_{c p}$ along the three principal axes for samples containing $60 \%$ aggregate particles volume fraction. Simulations were carried out at a global element size of $0.25 \mathrm{~mm}$.

\begin{tabular}{lcccc}
\hline & \multicolumn{4}{c}{ Realisation } \\
\cline { 2 - 5 } Diffusivity ratio & $\mathrm{i}$ & $\mathrm{Ii}$ & $\mathrm{iii}$ & $\mathrm{iv}$ \\
\hline$D_{x} / D_{c p}$ & 0.306 & 0.306 & 0.304 & 0.304 \\
$D_{y} / D_{c p}$ & 0.305 & 0.305 & 0.306 & 0.306 \\
$D_{z} / D_{c p}$ & 0.305 & 0.304 & 0.305 & 0.306 \\
\hline
\end{tabular}



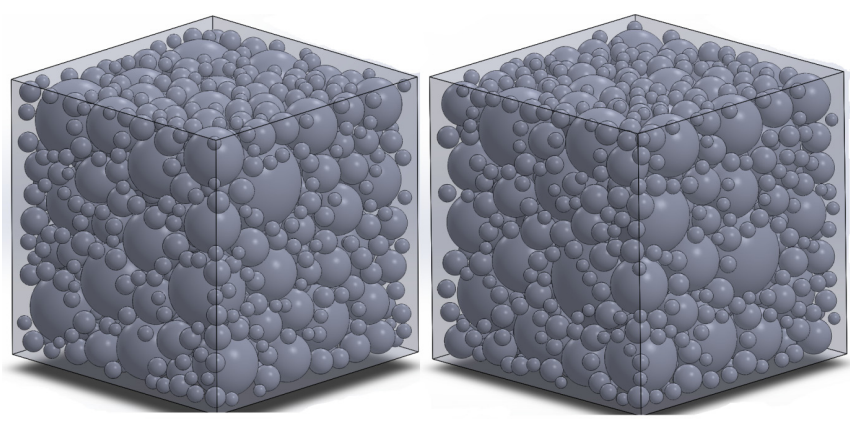

(a)

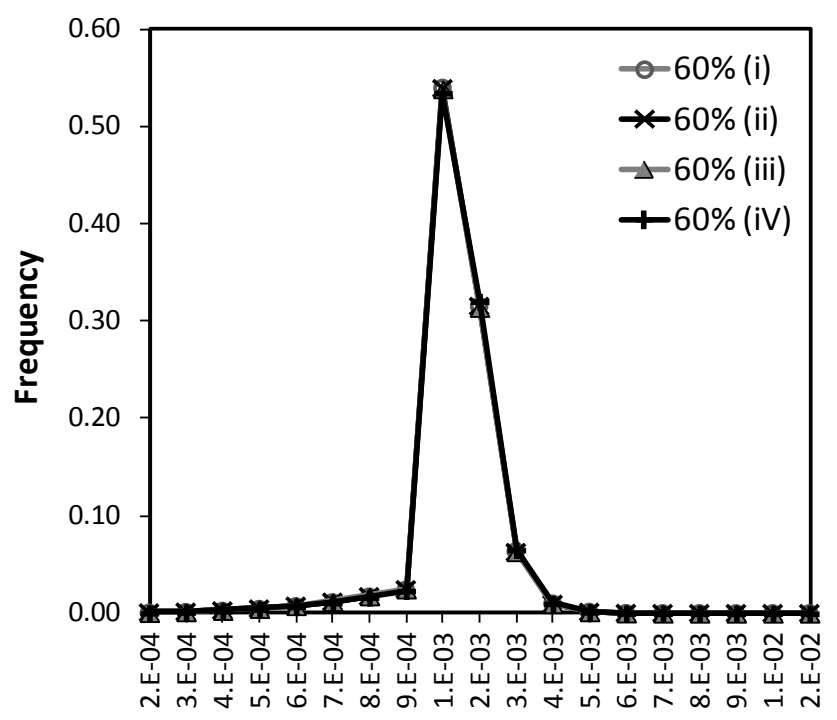

Element volume $\left(\mathrm{mm}^{3}\right)$

(b)
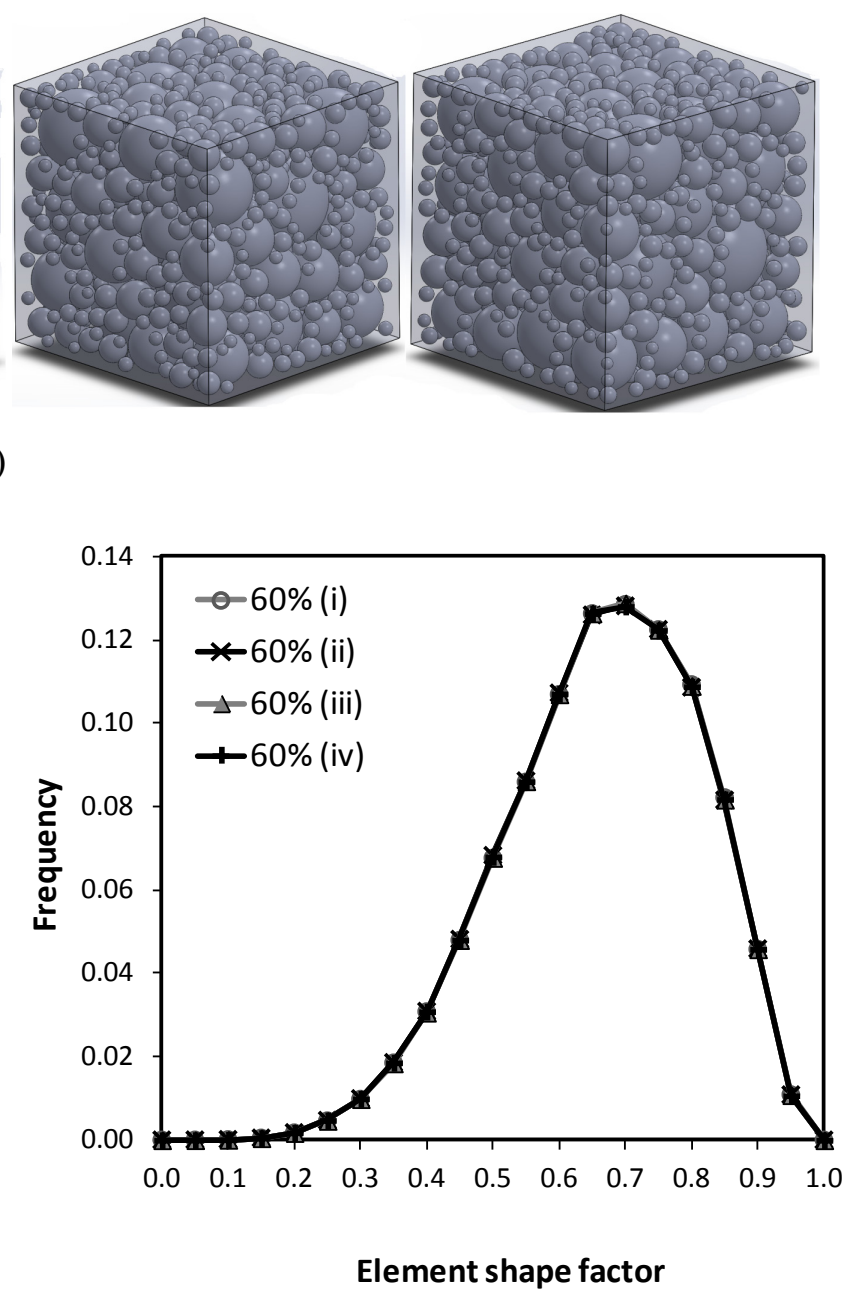

(c)

Fig. 7 Four different realisations of mesostructure containing $60 \%$ vol. aggregate particles with particles ranging from 1 to $10 \mathrm{~mm}$ (Fuller-Thompson gradation) in a $25 \times 25 \times 25 \mathrm{~mm}$ computational cube (a) and their respective frequency histograms for element volume (b) and shape factor (c).

\subsection{Comparison with Maxwell's equation}

Fig. 8 and Fig. 9 show the distribution of concentration, diffusion flux magnitude and diffusion flux in the $\mathrm{X}, \mathrm{Y}$ and $\mathrm{Z}$ directions for non-cracked concrete samples containing $10 \%$ and $60 \%$ vol. aggregate particles. The simulations were carried out at a global element size of $0.25 \mathrm{~mm}$. Concentrations equal to 100 and 10 were applied to the inlet and outlet respectively and the diffusivities of cement paste and aggregate particles were set arbitrarily to 1 and 0 respectively. It should be noted here that the concentration gradient and assigned diffusivity values do not affect final results as they will be reported in terms of ratio of diffusivity of cracked medium to uncracked medium. This is due to the fact that the governing differential equations are linear and consequently the results are independent of these input values and adopted units. 

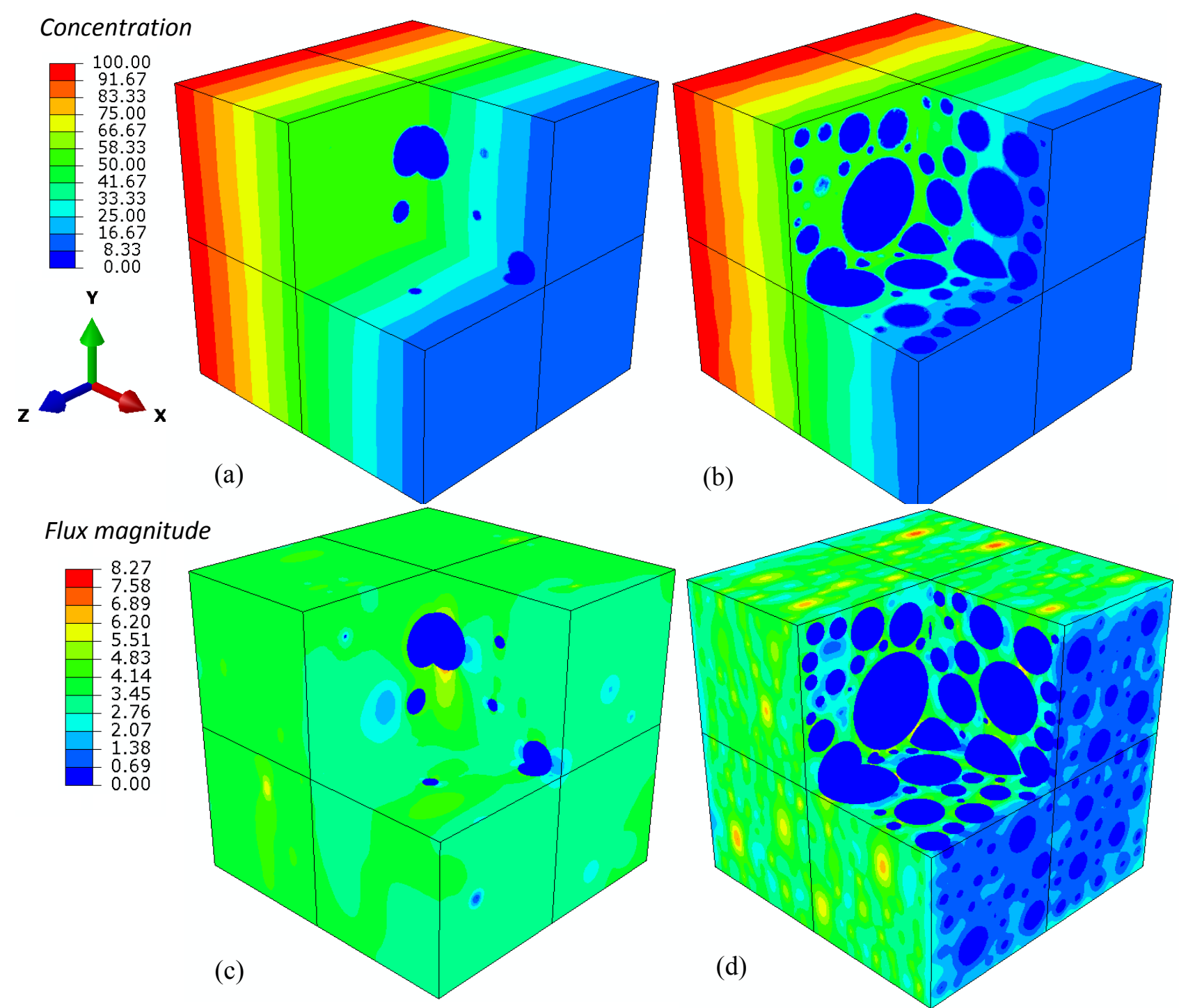

Fig. 8 Distribution of concentration (a, b) and flux magnitude (c, $d)$ in samples containing $10 \%$ and $60 \%$ vol. aggregate particles at steady-state condition. Concentrations equal to 100 and 10 are applied to the inlet and outlet respectively. The diffusivities of cement paste and aggregate particles are set to 1 and 0 respectively. 

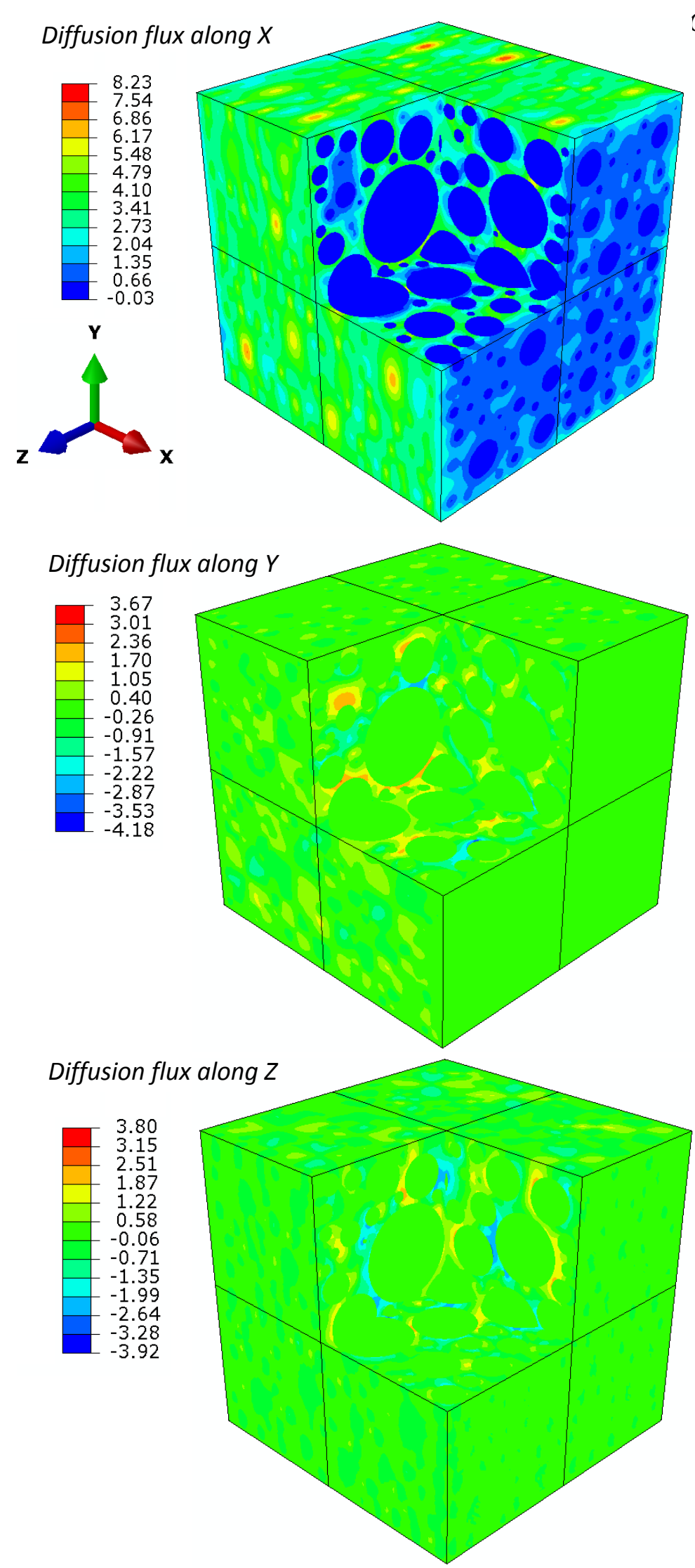

Fig. 9 Diffusion flux along the $X, Y$ and $Z$ direction in sample containing $60 \%$ vol. aggregate particles at steady-state condition. Concentrations equal to 100 and 10 are applied to the inlet and outlet respectively as shown in Fig. 7. The diffusivities of cement paste and aggregate particles are set to 1 and 0 respectively. 
As expected, the flux is primarily in one direction and the flux magnitudes at the inlet and outlet surfaces are smaller than the internal fluxes because of the reduction in area available to flow due to the presence of aggregate particles. Increasing aggregate particle volume fraction increases the difference between the flux in the inlet/outlet surface and internal sections. The results also show how the uniformity of the concentration distribution and the flux is changed by increasing aggregate particles volume fraction.

To check the accuracy of the numerical homogenisation, simulated diffusivities of concrete samples were compared with the classical Maxwell's approximation [Maxwell, 1954], which describes the macroscopic transport property of a binary composite containing spherical inclusions:

$$
\frac{D}{D_{c p}}=\frac{2 D_{c p}+D_{a}+2 V_{a}\left(D_{a}-D_{c p}\right)}{2 D_{c p}+D_{a}-V_{a}\left(D_{a}-D_{c p}\right)}
$$

Where $D$ is the effective diffusivity of the composite, $D_{c p}$ is the diffusivity of the continuous phase (cement paste matrix), and $D_{a}$ and $V_{a}$ are the diffusivity and volume fraction of the discrete inclusions (aggregate particles). Simulations were conducted on the samples with $10 \%, 30 \%, 50 \%$ and $60 \%$ vol. aggregate particles (Fig. 1) using a global element size of $0.25 \mathrm{~mm}$. As shown in Fig. 10, a very good agreement is observed between the simulated diffusivity and Maxwell's equation across a range of aggregate particle volume fraction and $D_{d} / D_{c p}$ ratios. The simulated values tend to be slightly smaller than those of Maxwell's equation and the error increases with increasing aggregate particle content. However, the largest percentage difference was only $0.6 \%$.

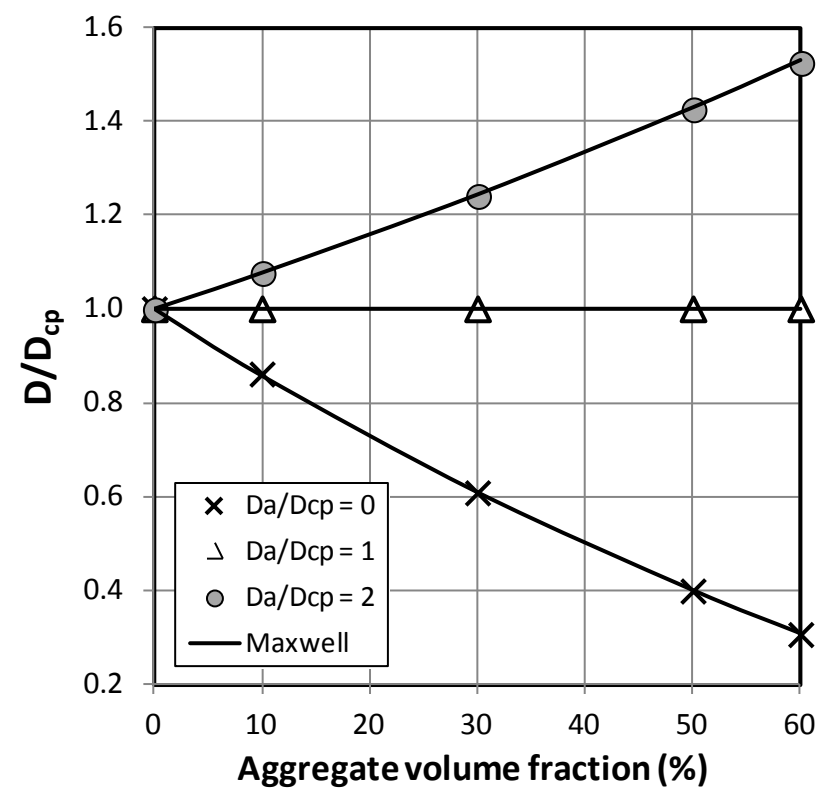

Fig. 10 Comparison between simulated diffusivity with Maxwell's equation for several aggregate particle volume fractions and $D_{d} / D_{c p}$ ratios.

\subsection{Samples containing parallel orthogonal microcracks}

An initial study of the effect of microcracks on transport was carried out by superimposing a simple parallel orthogonal microcrack network that traverses through the thickness of the mesostructure (Fig. 5a). Transport properties of the cracked sample were then simulated and the results compared to analytical solutions as a means of validation. For such a crack network, the equivalent diffusivity of the cracked sample $\left(D_{e q}\right)$ can be related to the crack characteristics such as width and diffusivity using a parallel model:

$$
D_{e q}=\frac{A_{c} D_{c}+\mathrm{AD}}{A_{c}+\mathrm{A}}
$$


Where $A_{C}$ is the area of the crack, $D_{C}$ is the diffusion coefficient of the crack, $A$ is the area of the uncracked medium and $D$ is the diffusion coefficient of the uncracked medium. Here, we assume that the diffusivity of the aggregate particles $\left(D_{a}\right)$ is zero. Although the crack diffusivity $D_{C}$ can be taken as the free diffusivity, the diffusivity of the uncracked medium will vary with porosity (w/c ratio, degree of hydration) and aggregate particle volume fraction. Therefore, simulations were conducted for several diffusivity contrast ratios $\left(D_{C} / D\right)$ of 100, 1000 and 10000, which should cover the range observed for most cement-based materials [Gerard et al. 2000, 1997]. Simulations were also carried out for several microcrack volume fractions ranging from $2 \%$ to $8 \%$.

Fig. 11 shows the simulated diffusivities compared with the analytical solution. The results show a very good agreement, thus giving confidence to our numerical model. It should be noted here that for the case of the parallel microcrack network, the cracks always form a percolated pathway. The microcrack volume fraction is the only influencing parameter while sample size, resolution and presence of aggregate particles do not influence the simulation. The diffusivity of the cracked medium increases and tends to infinity with increase in $D_{C} / D$ ratio.

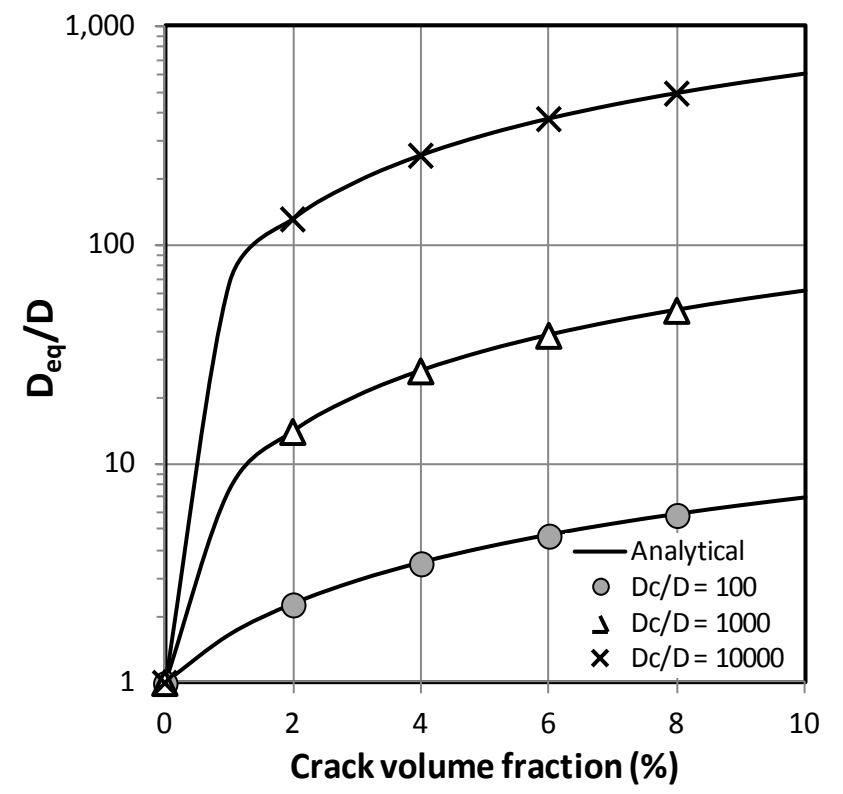

Fig. 11 Comparison between simulated diffusivity and analytical solution for samples containing parallel microcrack network (Fig. 5a) at several microcrack volume fractions and $D_{C} / D$ values.

\subsection{Samples containing bond microcracks}

A parallel crack network that traverses through the thickness of the sample would represent the worstcase scenario and yield an upper-bound diffusivity for the cracked medium. Another scenario is to consider microcracks occurring only at the aggregate-paste interface (Fig. 5 b, c). Here, the homogenisation can be carried out in two ways. The first is to homogenise the mesostructure containing aggregate particles and cement paste, and then incorporate microcracks in the homogenised medium [Gérard et al., 1997; Breysse \& Gérard, 1997]. The second approach is to incorporate microcracks as a third phase in a heterogeneous medium containing aggregate particles and cement paste (Fig. $5 \mathrm{c}$ ). In the simulations, microcrack volume fraction was increased by increasing the width of the bond microcracks from $1 \mu \mathrm{m}$ to $5,10,25$ and $50 \mu \mathrm{m}$. The simulations were also conducted for $D_{C} D$ ratios of 100,1000 and 10000 .

The results plotted in Fig. 12 show that diffusivity increases with microcrack volume fraction and $D_{c} / D$ ratio. Therefore, the bond microcracks can increase overall transport property despite the fact that the microcracks themselves are not percolated. However, the effect of bond microcracks on diffusivity is significantly smaller than that of the parallel orthogonal microcracks (Fig. 11). For the same microcrack volume fraction, the difference in diffusivity between the parallel crack network and bond crack network is very small at $D_{c} / D$ ratio of 100 . However, the difference increases with increase in $D_{c} / D$ ratio. At $D_{c} / D$ ratio of 1000, a difference of more than order of magnitude can be observed. This shows that when the microcracks are percolated, the effect on transport is highest in a denser medium (i.e. low w/c ratio, high 
degree of hydration) compared to a more porous medium. The results in Fig. 12 also show that placing microcracks in a homogenised medium (Fig. 5b) will lead to an incorrect and much smaller estimation of the effect of microcracks on overall transport property. Therefore, the approach for incorporating microcracks shown in Fig. $5 \mathrm{c}$ gives a better representation of a cracked mesostructure.

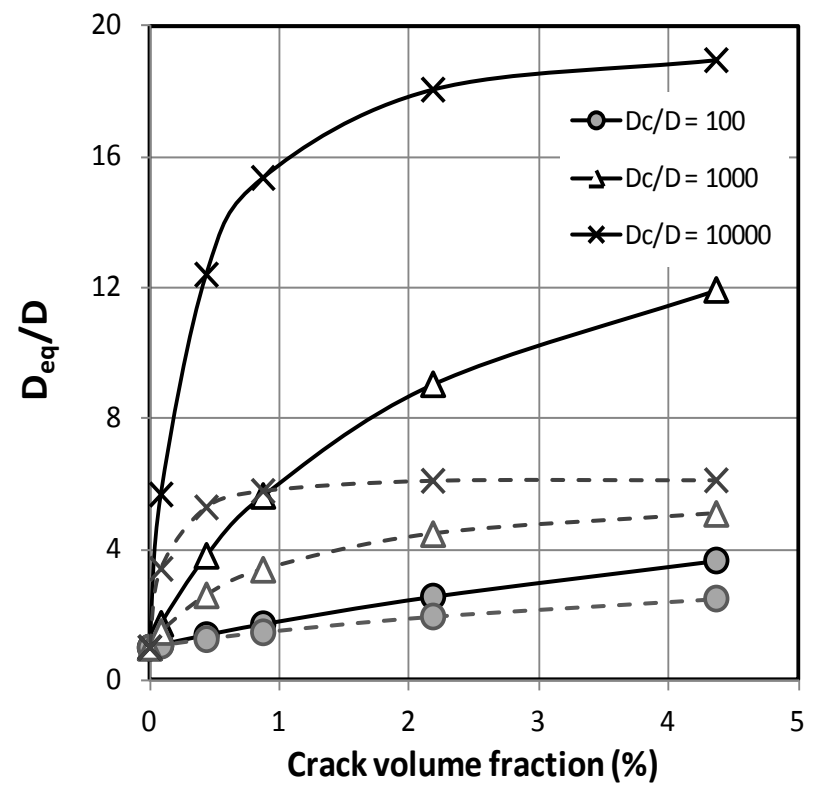

Fig. 12 Effect of bond microcracks on the diffusivity of concrete (60\% vol. aggregate particles). Dashed curves are for the case of bond microcracks dispersed in a homogenised media (Fig. 5b) and solid curves are for the case of bond microcracks dispersed in a heterogeneous media containing aggregate particles and cement paste (Fig. $5 \mathrm{c}$ ).

\subsection{Samples containing bond and matrix microcracks}

\subsubsection{Diffusivity}

Fig. 13 shows the effect of bond and matrix microcracks on the diffusivity of concrete containing $60 \%$ vol. aggregate particles. Note that the simulations were carried out for several microcrack patterns ( $a$ to $f$, Table 1). The simulations were also carried out at three contrast ratios, i.e. ratio of diffusivity of the microcrack to the diffusivity of uncracked concrete, of 100, 1000 and 10000 as in the earlier work. As expected, diffusivity increases with increase in microcrack volume fraction. For the same microcrack volume fraction, diffusivity increases with increase in the number of microcracks. Comparing the results to those shown in Fig. 12, it can be seen that the effect of microcrack networks containing only bond microcracks are of the same order as networks containing bond and matrix microcracks, when the latter are not percolated (case $a$ to $d$ ). Once the microcrack network becomes percolated (case $e \& f$ ), then diffusivity increases at a much higher rate compared to networks containing bond microcracks only, particularly at higher $D_{c} / D$ contrast ratios. 


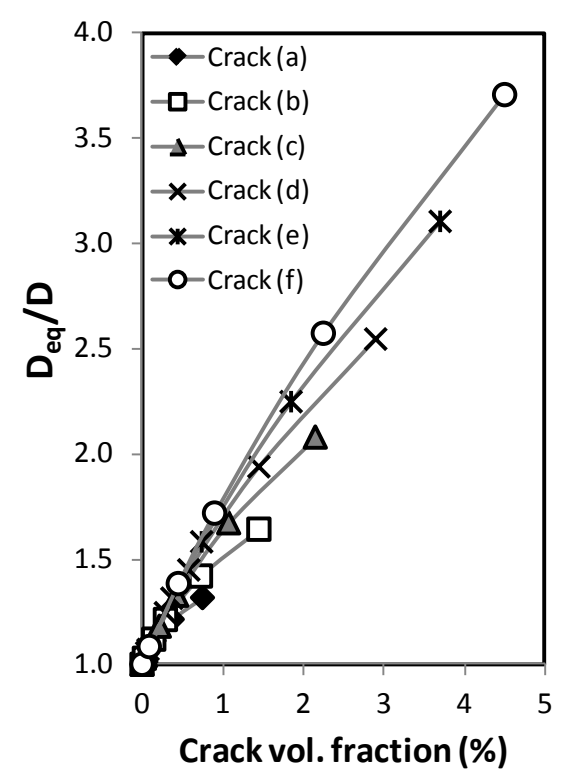

a) $D_{C} D=100$

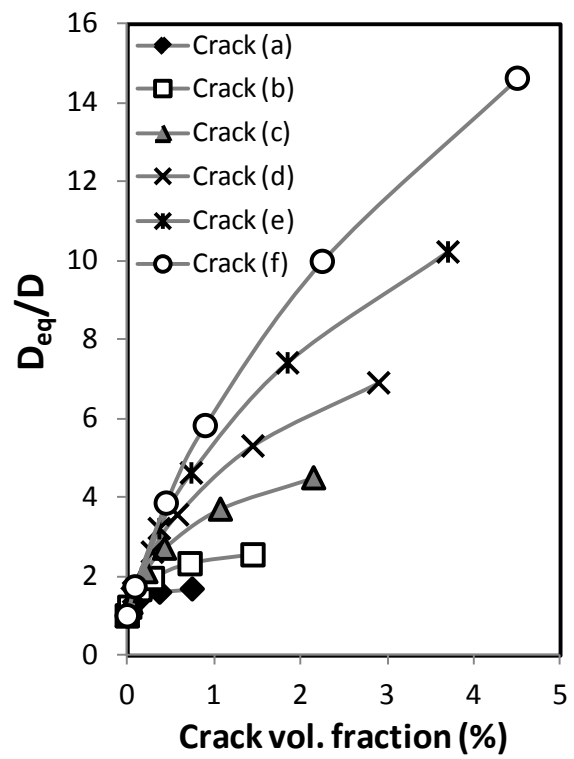

b) $D_{C} / D=1,000$

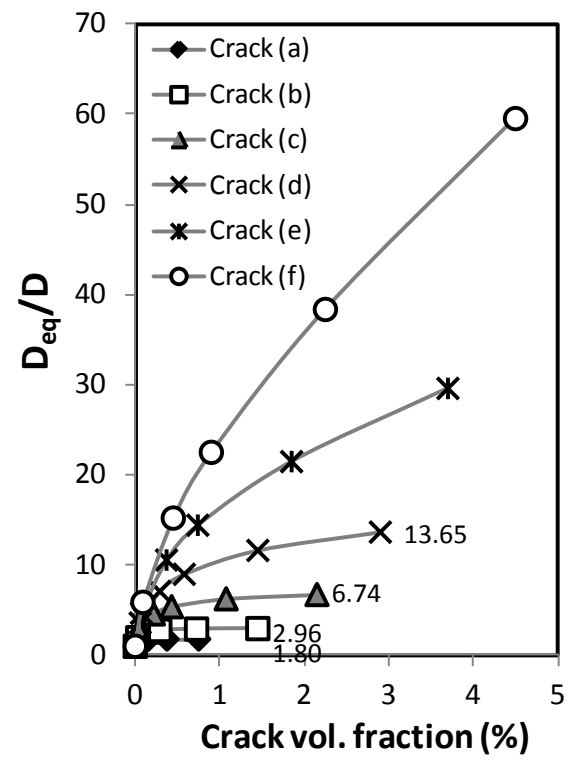

c) $D_{C} / D=10,000$

Fig. 13 Effect of matrix and bond microcracks on diffusivity of concrete $(60 \%$ vol. aggregate particles). Simulations were carried out for several microcrack patterns ( $a$ to $f$, Table 1$)$ and $D_{C} D$ contrast ratios.

Fig. 14 shows the effect of specific surface area of the microcracks on diffusivity as a function of microcrack width and contrast ratio. It can be seen that increasing the microcrack specific surface area of both non-percolated and percolated networks increases the equivalent diffusivity. This is because at higher specific surface area, the number of microcracks is increased while the distance that the diffusing species has to travel across the matrix is decreased. It is interesting to note that the change in diffusivity from a nonpercolated network (case $d$, specific surface $=0.58 \mathrm{~mm}^{2} / \mathrm{mm}^{3}$ ) to a percolated network (case $e$, specific surface $=0.74 \mathrm{~mm}^{2} / \mathrm{mm}^{3}$ ) is quite modest and not significantly different. This is in contrast with permeation which will be discussed in the next section. The results also show that the effect of contrast ratio is more pronounced for the microcrack networks with higher specific surface area.

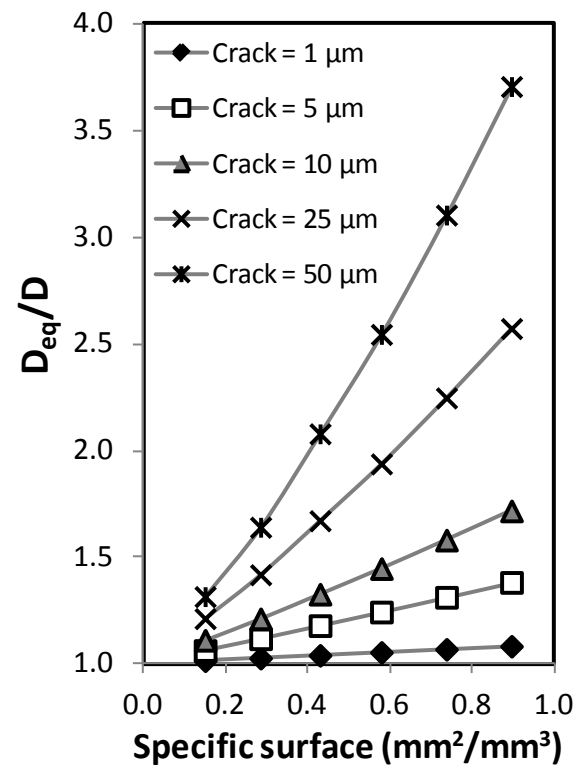

a) $D_{C} / D=100$

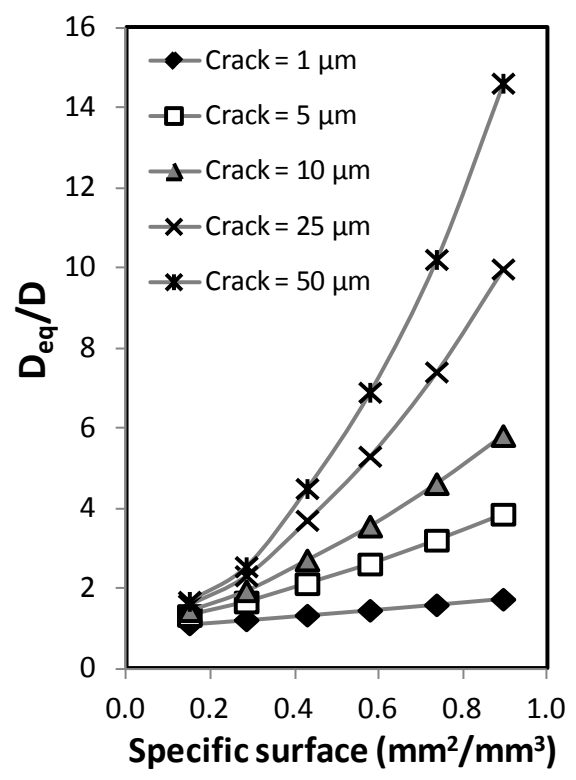

b) $D_{C} / D=1,000$

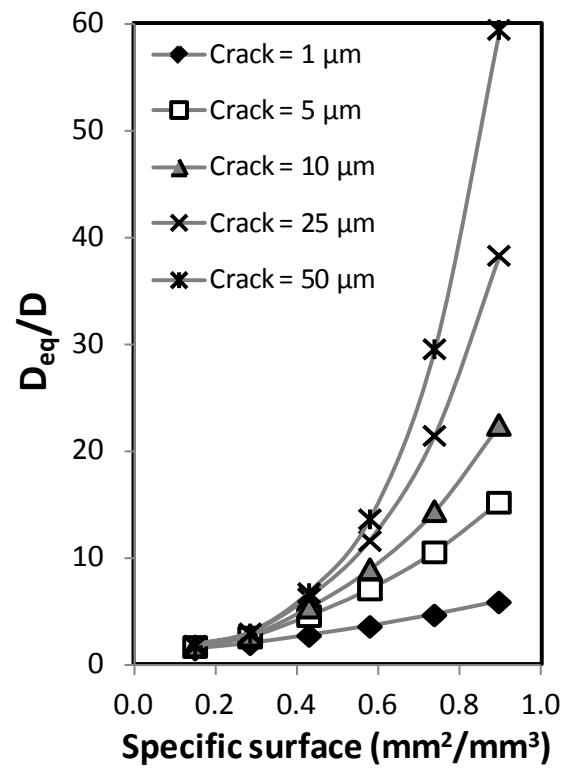

c) $D_{C} / D=10,000$ 
Fig. 14 Effect of specific surface area of microcracks on diffusivity of concrete $(60 \%$ vol. aggregate particles). Simulations were carried out for several microcrack patterns ( $a$ to $f$, Table 1$)$ and $D_{C} D$ contrast ratios.

\subsubsection{Permeability}

Figs. $15 \& 16$ show the effect of bond and matrix microcracks on the permeability of concrete containing $60 \%$ vol. aggregate particles. Simulations were carried out for microcrack patterns $a$ to $f$ (Table 1) and by assigning the permeability of uncracked concrete as either $1 \mathrm{E}-19,1 \mathrm{E}-18$ or $1 \mathrm{E}-17 \mathrm{~m}^{2}$, which covers the permeability of a wide range of concrete composition previously reported [Wong et al. 2009; Wu et al., 2015]. It should be noted that the approach here is slightly different to that used in the previous section where simulations were carried out by treating the ratio of diffusivity of microcrack to uncracked concrete as a variable. Here, simulations for permeation were carried out by treating the permeability of uncracked concrete as a variable. The reason for this is that permeability of a crack is a function of its width, while the diffusivity of a crack is equal to the free diffusivity and is independent of crack width.

Similar to the behaviour observed for diffusion, it can be seen that permeability increases with increase in microcrack volume fraction, number of microcracks and specific surface area. However, for the same microcrack volume fraction, the increase in permeability due to microcracks is much greater than that for diffusivity. The difference is greatest for percolated crack networks, where the increase in permeability is several orders of magnitude greater that the increase in diffusivity. Another interesting observation is that for non-percolated crack networks $(a, b, c \& d)$, the effective permeability increases with increase in microcrack volume fraction up to a finite limit (Fig. 15). The magnitude of this limit depends on characteristics of the microcrack network (e.g. number of bond and matrix microcracks, specific surface) and characteristics of the uncracked concrete (e.g. pore structure, aggregate particle content). In percolated networks however, this finite limit does not appear to exist and the effective permeability increases with increase in microcrack volume fraction.

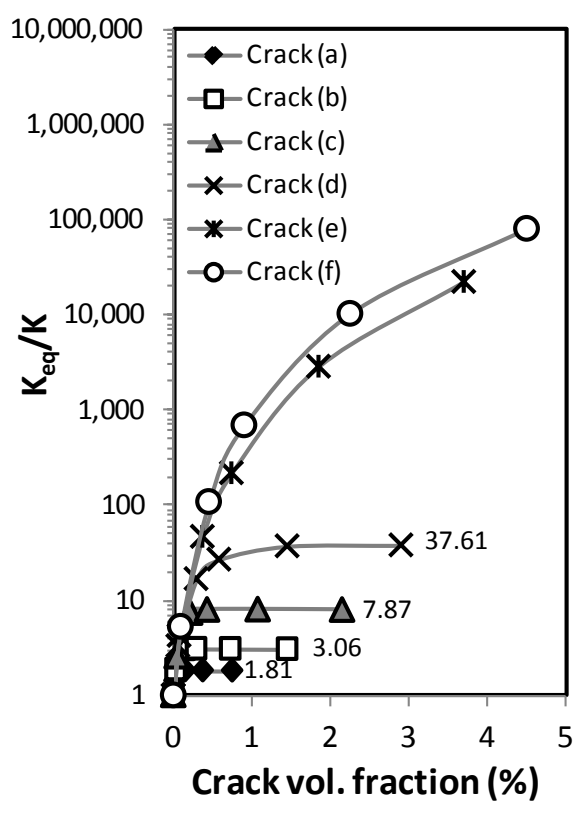

a) $K=1 \times 10^{-17} \mathrm{~m}^{2}$

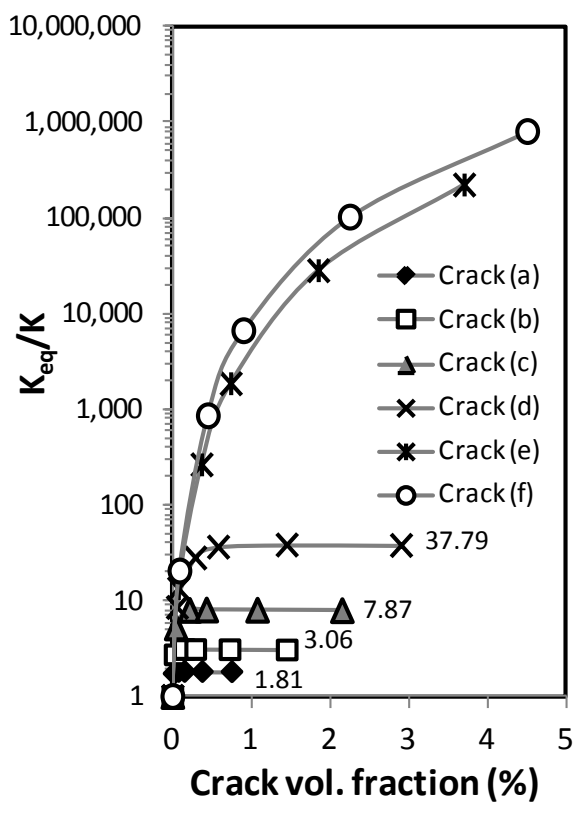

b) $K=1 \times 10^{-18} \mathrm{~m}^{2}$

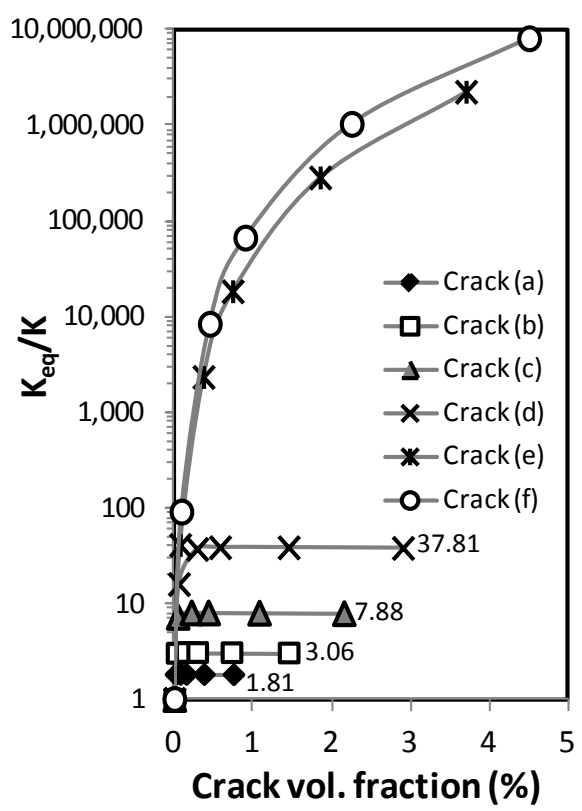

c) $K=1 \times 10^{-19} \mathrm{~m}^{2}$

Fig. 15 Effect of matrix and bond microcracks on permeability of concrete $(60 \%$ vol. aggregate particles). Simulations were carried out for several microcrack patterns ( $a$ to $f$, Table 1 ) and by assigning the permeability of the uncracked concrete $K$ as $1 \mathrm{E}-17$, $1 \mathrm{E}-18$ or $1 \mathrm{E}-19 \mathrm{~m}^{2}$.

The effect of percolation is more evident when the simulated permeability is plotted against specific surface area in Fig. 16, where a sudden large increase in permeability between the fourth and fifth data point 
can be seen. This corresponds to the situation where a non-percolated network (case $d$, specific surface $=$ $0.58 \mathrm{~mm}^{2} / \mathrm{mm}^{3}$ ) changes to a percolated network (case $e$, specific surface $=0.74 \mathrm{~mm}^{2} / \mathrm{mm}^{3}$ ). The jump in permeability becomes more severe for larger microcrack widths and for denser matrix. It should be recalled that this behaviour was not observed in the case of diffusion.

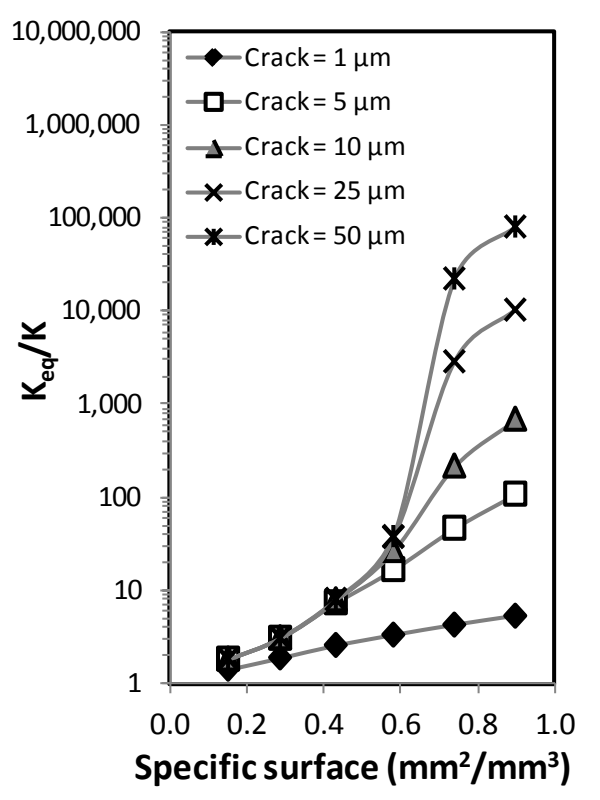

a) $K=1 \times 10^{-17} \mathrm{~m}^{2}$

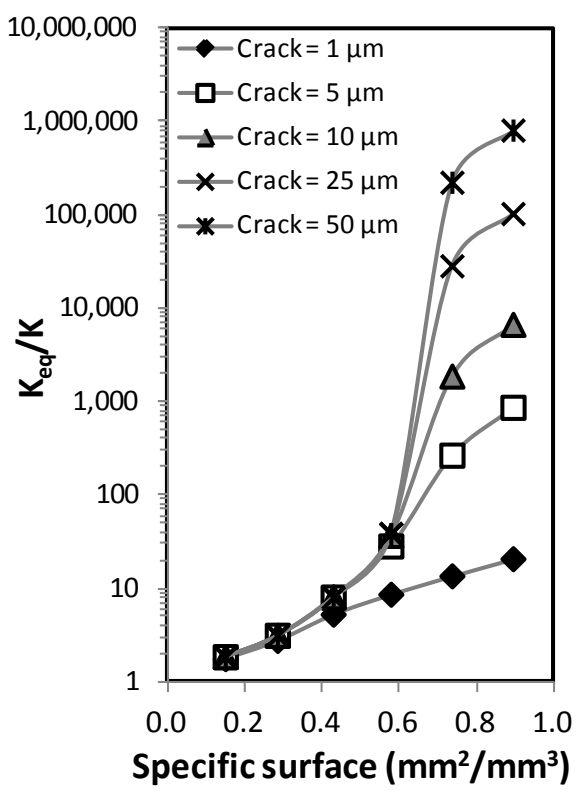

b) $K=1 \times 10^{-18} \mathrm{~m}^{2}$

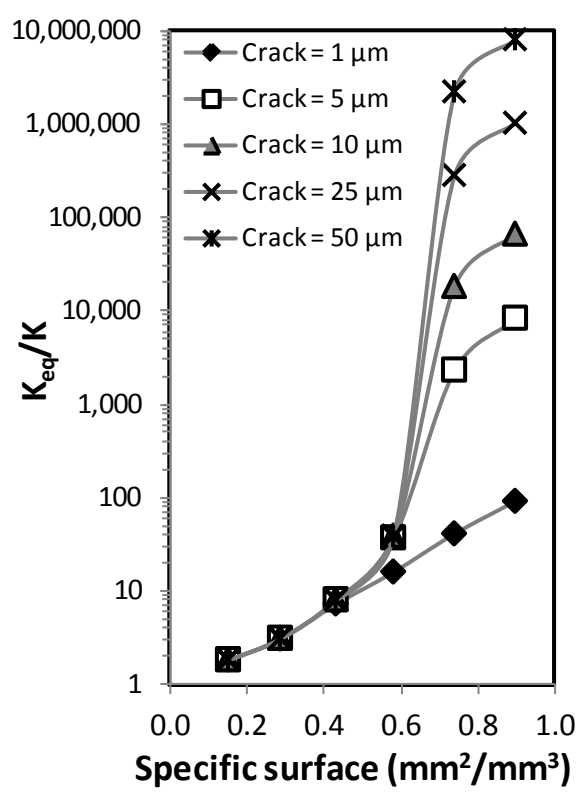

c) $K=1 \times 10^{-19} \mathrm{~m}^{2}$

Fig. 16 Effect of specific surface area of microcracks on permeability of concrete $(60 \%$ vol. aggregate particles). Simulations were carried out for several microcrack patterns ( $a$ to $f$, Table 1$)$ and by assigning the permeability of the uncracked concrete $K$ as $1 \mathrm{E}-17$, $1 \mathrm{E}-18$ or $1 \mathrm{E}-19 \mathrm{~m}^{2}$.

\section{Discussion}

The simulations show that the effect of microcracks on transport properties is more pronounced on materials with a denser matrix, for example, concretes with low water to cement ratio or high degree of hydration, compared to a more porous matrix. These materials would have higher $D_{c} / D$ diffusivity contrast ratios and lower permeability in the uncracked state. The main parameters influencing the effect of microcracks on the transport properties of concrete are the size, specific surface area, interconnectivity and degree of percolation of the microcrack network. In general, the effect of microcracks is more pronounced for permeation compared to diffusion, but the extent of the difference depends on many variables.

For a non-percolating microcrack network, the effective transport property of the cracked concrete increases with microcrack volume fraction initially, but reaches a limit beyond a certain microcrack volume fraction and remains relatively constant thereafter. The magnitude of this limit depends on the characteristics of the microcrack network and porous matrix. This finite limit exists because in non-percolated microcrack networks, flux has to travel across the uncracked cement paste and so the effective transport property is governed by the transport property of the uncracked matrix. For example, comparing Fig. 13c and Fig. 15, it can be seen that for a similar non-percolated microcrack network $(a, b, c$ and $d)$, the "finite limit" for diffusion and permeation are nearly the same in magnitude. However, this limit is reached much earlier (at lower microcrack volume fraction) for the case of permeation.

The connectivity of a microcrack network and the point at which it percolates would depend on the number and distribution of the bond and matrix microcracks, their lengths and therefore their specific surface area. At higher specific surface area, there are more microcracks present, the separation distance between microcracks decreases and the distance that the diffusing species has to travel across the cement paste matrix decreases. Therefore, increasing the specific surface area increases the equivalent diffusivity and permeability for both non-percolated and percolated microcrack networks. 
When the microcrack network becomes percolated, fluid flows predominantly through the microcracks and so the effective transport property now increases beyond this limit as it is governed by the transport property of the microcrack. For the case of diffusion, this will be the free diffusion coefficient of the species under consideration, while for the case of pressure-induced flow, the permeability of the crack which increases with crack width. This means that for sparsely populated microcrack networks that are not percolated, the effect of microcrack is of the same order for diffusion and permeation. However, in densely populated microcrack networks that are percolated, the effect becomes significantly more pronounced for permeation compared to diffusion. This can be also be deduced by comparing Fig. 14 and Fig. 16. A massive jump in permeability is seen when the microcrack network becomes percolated (at specific surface area $>0.6$ $\mathrm{mm}^{2} / \mathrm{mm}^{3}$ ), while the increase in diffusivity is comparatively modest.

However, the microstructure of real concrete is obviously more complicated than what is captured by the three-dimensional meso scale model presented in this paper. In practice concrete has slightly higher aggregate particle volume fraction $(>60 \%)$ and wider particle size range than the sample simulated in this study. The smallest aggregate particles considered were $1 \mathrm{~mm}$ due to the limitations of the mesh generator and computational resources. However, it should be noted that small aggregate particles have less contribution to microcracking as observed in previous studies [Bisschop and van Mier, 2002; Idiat et al., 2012; Wu et al., 2015]. These studies showed that the degree of microcracking (measured in terms of width, length, depth or density) decreases with decrease in aggregate particle size. Furthermore, fracture mechanics predict that aggregate particles below a critical size do not cause microcracking [Goltermann, 1994]. Here, we assume the critical size is $1 \mathrm{~mm}$.

It is also well-known that the presence of aggregate particles disturbs the microstructure of the cement paste. This causes the cement paste surrounding each aggregate particle, i.e. the 'interfacial transition zone' (ITZ), to contain on average higher porosity than the 'bulk' cement paste farther away. Therefore, the transport property of the ITZ should be on average higher than the bulk cement paste, but this is known to be spatially variable. Such effects were not considered in the simulations presented in this study for the sake of simplicity and because we were mainly interested in quantifying the effects of microcracking. Furthermore, the effect of ITZ on transport properties is very small relative to the effect of microcracks, as shown in our previous experimental and numerical studies [Wong et al., 2009; Abyaneh et al., 2013a] and more recently in [Bernard \& Kamali-Bernard, 2015]. Therefore, the findings of this study are not expected to be substantially influenced by this approximation since the effect of the added microcracks would be far greater than that of the ITZ. Indeed, the order of magnitude increase in transport property simulated in this study is well within the range of those reported in the literature [Jacobsen et al. 1996; Djerbi et al., 2008; Wong et al., 2009; Kamali-Bernard and Bernard, 2009; Wu et al., 2015]

Ideally our simulated results would be compared with experimental measurements. However, the availability of such experimental data that are suitable for direct comparison is at best scarce and probably non-existent. Although a number of researchers have conducted mass transport experiments on cracked concrete, most have only reported the measured transport property and do not provide information on the microstructure, in particular the characteristics of the microcrack network. Where measurements of microcracks are available, these are generally carried out on two-dimensional cross sections and so the critical information on the way in which the microcracks are interconnected is missing. This issue is compounded by the lack of suitable techniques to characterise a representative volume of concrete in threedimensions at a sufficient resolution to capture the microcracks. There are also issues with transport measurements. For example, some of the microcracks will probably self heal when exposed to moisture for prolonged periods as required in many types of transport experiments. However, our simulations have not considered chemical reactions such as those associated with hydration, leaching and microcrack healing.

\section{Conclusions}

This study proposes a finite-element approach for three-dimensional simulations to study the effect of microcracks $(1-50 \mu \mathrm{m})$ on the diffusivity and permeability of concrete containing $60 \%$ vol. aggregate particles ranging from 1 to $10 \mathrm{~mm}$ in size. To improve computational efficiency, an aligned meshing approach was applied where discretisation was carried out using tetrahedral elements and triangulation to explicitly capture the surfaces of aggregate particles. Microcracks were then incorporated as interface elements, enabling the smallest microcracks to be represented independent of the size of the discretisation of 
the aggregate particles and cement paste. The model was first validated by comparing with analytical solutions for simple/ideal mesostructures and microcrack geometries (e.g. parallel orthogonal microcrack networks). Then, the model was applied to carry out a sensitivity analysis on concrete samples containing several microcrack patterns consisting of bond microcracks at the aggregate-paste interface and matrix microcracks spanning nearest neighbouring aggregate particles. The samples considered included a wide range of characteristics such as microcrack widths (1 to $50 \mu \mathrm{m}$ ), number of individual microcracks (350 to 2050), microcrack volume fractions (0.02 to 5.4\%), specific surface area $\left(0.15\right.$ to $\left.0.90 \mathrm{~mm}^{2} / \mathrm{mm}^{3}\right)$, degree of percolation, diffusivity contrast ratio $\left(D_{c} / D=100\right.$ to 10000$)$ and permeability $\left(1 \mathrm{E}-19\right.$ to $\left.1 \mathrm{E}-17 \mathrm{~m}^{2}\right)$. The main conclusions are:

a) The effect of microcracks on diffusion and permeation is more pronounced for concretes with denser matrix (e.g. low w/c ratio, high degree of hydration) compared to a more porous matrix.

b) For concretes containing sparsely populated microcrack networks that are not percolated, the effective transport property of the cracked concrete increases with microcrack volume fraction up to a finite limit because flow is governed by the transport property of the uncracked matrix. The limiting value depends on the characteristics of the microcrack network (e.g. number of bond and matrix microcracks, specific surface) and uncracked concrete (e.g. pore structure, aggregate particle content). The limiting value is reached much earlier at lower microcrack volume fraction for the case of permeation compared to diffusion.

c) For concretes containing densely populated microcrack networks that are percolated, the effective transport property increases continuously with increase in microcrack volume fraction since flow is now governed by the transport property of microcracks. For diffusion, this is the free diffusivity of the species under consideration, and for pressure-induced flow, the permeability of the microcrack which increases with crack width.

d) Increasing microcrack specific surface area increases the equivalent transport property for both nonpercolated and percolated microcrack networks. This is because at higher specific surface area, there are more microcracks present and the distance that the transporting species has to travel across the uncracked matrix is decreased.

e) In all cases, the presence of microcracks has a greater effect on permeation compared to diffusion. The magnitude of difference becomes more pronounced in percolated microcrack networks. This can be seen in the sudden large surge in permeability when the microcracks become percolated.

f) The modelling approach described in this paper is particularly useful for evaluating the effect of various parameters, such as microcracks, on transport properties, where laboratory experimentation alone would be unable to isolate and quantify the effect.

\section{Acknowledgements}

The research leading to these results has received funding from the European Union Seventh Framework Programme (FP7 / 2007-2013) under grant agreement 264448.

\section{References}

Abyaneh, S.D., Wong, H.S., Buenfeld, N.R. (2015), Modelling the effect of microcracks on the transport properties of concrete in three-dimensions, CONCREEP-10 Proceedings, 377-386

Abyaneh, S.D., Wong, H.S., Buenfeld, N.R. (2013a). Modelling the diffusivity of mortar and concrete using a three-dimensional mesostructure with several aggregate shapes, Computational Materials Science, 78, 6373.

Abyaneh, S.D., Wong, H.S., Buenfeld, N.R. (2013b). Modelling the transport properties of concrete from three-dimensional mesostructure, TRANSCEND Conference, 3-6 November, Guildford, Surrey.

Abyaneh, S.D., Wong, H.S., Buenfeld, N.R. (2014), Computational investigation of capillary absorption in concrete using a three-dimensional mesoscale approach, Computational Materials Science, 87, 54-64. 
Adler, P. M., Thovert, J.-F. O., Mourzenko, V. V. (2012). Fractured porous media, Oxford, Oxford University Press.

Aldea, C. M., Shah, S. P., Karr, A. (1999). Permeability of cracked concrete, Mater. Struct., 32, 370-376.

Akhavan, A., Shafaatian, S.-M.-H., Rajabipour, F. (2012). Quantifying the effects of crack width, tortuosity, and roughness on water permeability of cracked mortars, Cem. Concr. Res., 42, 313-320.

Bernard, F., Kamali-Bernard, S. (2015). Numerical study of ITZ contribution on mechanical behavior and diffusivity of mortars. Computational Materials Science, 102, 250-257.

Bisschop, J., van Mier, J.G.M. (2002). Effect of aggregates on drying shrinkage microcracking in cementbased composites, Mater. Struct. 35, 453-461.

Bogdanov, I. I., Mourzenko, V. V., Thovert, J. F. \& Adler, P. M. (2003). Effective permeability of fractured porous media in steady state flow. Water Resources Research, 39. 1023

Breysse, D., Gérard, B. (1997). Transport of fluids in cracked media. In: Reinhardt, H. W. (ed.) RILEM report no. 16: Penetration and Permeability of Concrete: Barriers to Organic and Contaminating Liquids. London.

Concrete Society (1995), Technical Report 44: The relevance of cracking in concrete corrosion of reinforcement, Camberley.

Concrete Society (2010), Technical Report 22: Non-structural cracks in concrete, 4th Edition, Camberley.

Djerbi, A., Bonnet, S., Khelidj, A. \& Baroghel-Bouny, V. (2008). Influence of traversing crack on chloride diffusion into concrete. Cement and Concrete Research, 38, 877-883.

Djerbi, A., Bonnet, S., Khelidj, A. \& Baroghel-Bouny, V. (2013). Effect of uniaxial compressive loading on gas permeability and chloride diffusion coefficient of concrete and their relationship, Cem. Concr. Res., 52, 131-139.

Du, X., Jin, L. \& Ma, G. (2014). A meso-scale numerical method for the simulation of chloride diffusivity in concrete, Finite Elements in Analysis and Design., 85, 87-100.

Feng, G-L., Li, L-Y., Kim, B. \& Liu, Q-F. (2016). Multiphase modelling of ionic transport in cementitious materials with surface charges, Computational Materials Science., 111, 339-349.

Garboczi, E. J., Bentz, D. P. (2001). The effect of statistical fluctuation, finite size error, and digital resolution on the phase percolation and transport properties of the NIST cement hydration model. Cem. Concr. Res., 31, 1501-1514.

Gérard, B., Reinhardt, H. W., Breysse, D. (1997). Measured transport in cracked concrete. In: Reinhardt, H. W. (ed.) RILEM Report 16: Penetration and Permeability of Concrete: Barriers to Organic and Contaminating Liquids. London.

Gérard, B., Marchand, J. (2000). Influence of cracking on the diffusion properties of cement-based materials: Part I: Influence of continuous cracks on the steady-state regime. Cem Concr Res, 30, 37-43.

Goltermann, P. (1994). Mechanical predictions on concrete deterioration. Part 1: Eigenstresses in concrete. ACI Materials Journal, 91, 543-550.

Goltermann, P. (1995). Mechanical predictions of concrete deterioration - Part 2: classification of crack patterns. ACI Materials Journal, 92, 58-63.

Grassl, P., Wong, H. S. \& Buenfeld, N. R. (2010). Influence of aggregate size and volume fraction on shrinkage induced micro-cracking of concrete and mortar. Cem. Concr. Res., 40, 85-93.

Hsu, T. T. C. (1963). Mathematical analysis of shrinkage stresses in a model of hardened concrete. ACI Journal, 60 [3] 371-389.

Idiart, A., Bisschop, J., Caballero, A., Lura, P. (2012). A numerical and experimental study of aggregateinduced shrinkage cracking in cementitious composites. Cem. Concr. Res. 42, 272-281.

Jacobsen, S. , Marchand, J., Boisvert, L. (1996). Effect of cracking and healing on chloride transport in OPC concrete. Cem. Concr. Res., 26, 869-881. 
Jang, S. Y., Kim, B. S. \& Oh, B. H. (2011). Effect of crack width on chloride diffusion coefficients of concrete by steady-state migration tests. Cem. Concr. Res. 41, 9-19.

Kamali-Bernard, S. \& Bernard, F. (2009). Effect of tensile cracking on diffusivity of mortar: 3D numerical modelling. Computational Materials Science, 47, 178-185.

Liu, Q-F., Yang, J., Xia, J., Easterbrook, D., Li, L-Y. \& Lu, X-Y (2015). A numerical study on chloride migration in cracked concrete using multi-component ionic transport models, Computational Materials Science, 99, 396-416.

Maxwell, J.C. (1954), A treatise on electricity and magnetism, Vol. 1 \& 2, Dover Publications, New York.

Moreno-Atanasio, R., Williams, R. A. \& Jia, X. (2010). Combining X-ray microtomography with computer simulation for analysis of granular and porous materials, Particuology, 8, 81-99.

Picandet, V., Khelidj, A. \& Bellegou, H. (2009). Crack effects on gas and water permeability of concretes. Cem. Concr. Res., 39, 537-547.

Sahimi, M. 2011. Flow and transport in porous media and fractured rock : from classical methods to modern approaches, Weinheim, Wiley-VCH.

Slate, F. O., Olsefski, S. (1963). X-Rays for study of internal structure and microcracking of concrete. ACI Journal Proceedings, 60, 575-588.

Shah, S. P., Chandra, S. (1968). Critical stress, volume change, and microcracking of concrete. ACI Journal Proceedings, 65, 770-780.

Stauffer, D. (1985), Introduction to Percolation Theory, Taylor \& Francis, London.

Wang, K. J., Jansen, D. C., Shah, S. P. \& Karr, A. F. (1997). Permeability study of cracked concrete. Cem. Concr. Res. 27, 381-393.

Wong, H. S., Zobel, M., Buenfeld, N. R., Zimmerman, R. W. (2009). Influence of the interfacial transition zone and microcracking on the diffusivity, permeability and sorptivity of cement-based materials after drying. Magazine of Concrete Research, 61, 571-589.

Wu, Z., Wong, H. S., Buenfeld, N. R. (2014). Effect of confining pressure and microcracks on mass transport properties of concrete. Advances in Applied Ceramics, 113, 485-495.

Wu, Z., Wong, H. S., Buenfeld, N. R. (2015). Influence of drying-induced microcracking and related size effects on mass transport properties of concrete. Cem. Concr. Res., 68, 35-48.

Zalzale, M., Mcdonald, P. J. (2012). Lattice Boltzmann simulations of the permeability and capillary adsorption of cement model microstructures. Cem. Concr. Res., 42, 1601-1610.

Zhang, M., Ye, G., Breugel, K. V. (2013). Microstructure-based modeling of permeability of cementitious materials using multiple-relaxation-time lattice Boltzmann method. Computational Materials Science, 68, 142-151. 